\title{
DISCRETE MECHANICS AND OPTIMAL CONTROL: AN ANALYSIS*
}

\author{
Sina Ober-Blöbaum ${ }^{1}$, Oliver Junge ${ }^{2}$ and Jerrold E. Marsden ${ }^{3}$
}

\begin{abstract}
The optimal control of a mechanical system is of crucial importance in many application areas. Typical examples are the determination of a time-minimal path in vehicle dynamics, a minimal energy trajectory in space mission design, or optimal motion sequences in robotics and biomechanics. In most cases, some sort of discretization of the original, infinite-dimensional optimization problem has to be performed in order to make the problem amenable to computations. The approach proposed in this paper is to directly discretize the variational description of the system's motion. The resulting optimization algorithm lets the discrete solution directly inherit characteristic structural properties from the continuous one like symmetries and integrals of the motion. We show that the DMOC (Discrete Mechanics and Optimal Control) approach is equivalent to a finite difference discretization of Hamilton's equations by a symplectic partitioned Runge-Kutta scheme and employ this fact in order to give a proof of convergence. The numerical performance of DMOC and its relationship to other existing optimal control methods are investigated.
\end{abstract}

Mathematics Subject Classification. 49M25, 49N99, 65K10.

Received October 8, 2008. Revised September 17, 2009.

Published online March 31, 2010.

\section{INTRODUCTION}

In order to solve optimal control problems for mechanical systems, this paper links two important areas of research: optimal control and variational mechanics. The motivation for combining these fields of investigation is twofold. Besides the aim of preserving certain properties of the mechanical system for the approximated optimal solution, optimal control theory and variational mechanics have their common origin in the calculus of variations. In mechanics, the calculus of variations is also fundamental through the principle of stationary action; that is, Hamilton's principle. When applied to the action of a mechanical system, this principle yields the equations of motion for that system - the Euler-Lagrange equations. In optimal control theory the calculus of variations also plays a fundamental role. For example, it is used to derive optimality conditions via the Pontryagin maximum principle. In addition to its importance in continuous mechanics and control theory, the discrete calculus of variations and the corresponding discrete variational principles play an important role in constructing efficient numerical schemes for the simulation of mechanical systems and for optimizing dynamical systems.

\footnotetext{
Keywords and phrases. Optimal control, discrete mechanics, discrete variational principle, convergence.

* Research partially supported by the University of Paderborn, Germany and AFOSR grant FA9550-08-1-0173.

1 Department of Mathematics, Faculty of Electrical Engineering, Computer Science and Mathematics, University of Paderborn, 33098 Paderborn, Germany. sinaob@math.upb.de

2 Zentrum Mathematik, Technische Universität München, 85747 Garching, Germany. junge@ma.tum.de

3 Control and Dynamical Systems, California Institute of Technology 107-81, Pasadena, CA 91125, USA.

jmarsden@caltech.edu
} 
Discrete optimal control and discrete variational mechanics. The theory of discrete variational mechanics has its roots in the optimal control literature of the 1960s; see for example [14,33,34]. Specifically, [14] developed a discrete calculus of variations theory in the following way: A function is introduced which depends on a sequence of numbers, e.g. a sequence of times. A minimizing sequence necessarily satisfies a second-order difference equation, which is called the discrete Euler equation in reminiscence of its similarity with the Euler equation of the classical calculus of variations. An application of the discrete calculus of variations to an optimal control problem leads to a so called direct solution method. In this, one transforms the optimal control problem into a finite dimensional equality constrained nonlinear optimization problem via a finite dimensional parameterization of states and controls. In contrast, indirect methods (see Sect. 2.3 for an overview) are based on the explicit derivation of the necessary optimality conditions via the Pontryagin maximum principle.

On the other hand, the theory of discrete variational mechanics describes a variational approach to discrete mechanics and mechanical integrators. The application of a discrete version of Hamilton's principle results in the discrete Euler-Lagrange equations. Analogous to the continuous case, near conservation of discrete energy, discrete momentum maps related to the discrete system's symmetries and the discrete symplectic form can be shown. This is due to the discretization of the variational structure of the mechanical system directly. Early work on discrete mechanics was often independently done by $[15,50,51,58,60-62]$. In this work, the role of the discrete action sum, the discrete Euler-Lagrange equations and the discrete Noether's theorem were clearly understood. The variational view of discrete mechanics and its numerical implementation is further developed in $[85,86]$ and then extended in $[5,6,38,39,66,67]$. The route of a variational approach to symplectic-momentum integrators has been taken by [59,80]; see the review by [64] and references therein. In this review a detailed derivation and investigation of these variational integrators for conservative as well as for forced and constrained systems is given.

Combining optimal control and variational mechanics. The present paper concerns the optimal control of dynamical systems whose behavior can be described by the Lagrange-d'Alembert principle. To numerically solve this kind of problem, we make use of the discrete calculus of variations only, that means we apply the discrete variational principle on two layers. On the one hand we use it for the description of the mechanical system under consideration, and on the other hand for the derivation of necessary optimality conditions for the optimal control problem. The application of discrete variational principles already on the dynamical level (namely the discretization of the Lagrange-d'Alembert principle) leads to structure-preserving time-stepping equations which serve as equality constraints for the resulting finite dimensional nonlinear optimization problem. The benefits of variational integrators are handed down to the optimal control context. For example, in the presence of symmetry groups in the continuous dynamical system, also along the discrete trajectory the change in momentum maps is consistent with the control forces. Choosing the objective function to represent the control effort, which has to be minimized is only meaningful if the system responds exactly according to the control forces.

Related work. A survey of different methods for the optimal control of dynamical systems described by ordinary differential equations is given in Section 2.3. However, to our knowledge, DMOC is the first approach to solutions of optimal control problems involving the concept of discrete mechanics to derive structure-preserving schemes for the resulting optimization algorithm. Since our first formulations and applications to space mission design and formation flying [35-37,71], DMOC has been applied for example to problems from robotics and biomechanics $[40,46,47,68,73,78]$ and to image analysis [69]. From the theoretical point of view, considering the development of variational integrators, extensions of DMOC to mechanical systems with nonholonomic constraints or to systems with symmetries are quite natural and have already been analyzed in [46,47]. Further extensions are currently under investigation, for example DMOC for hybrid systems [73] and for constrained multi-body dynamics (see $[56,57,71]$ ). DMOC related approaches are presented in $[52,53]$. The authors discretize the dynamics by a Lie group variational integrator. Rather than solving the resulting optimization problem numerically, they construct the discrete necessary optimality conditions via the discrete variational principle and solve the resulting discrete boundary value problem (the discrete state and adjoint system). The method is applied to the optimal control of a rigid body and to the computation of attitude maneuvers of a rigid spacecraft. 
Outline. In Sections 1.1 and 1.2 we introduce the relevant concepts from classical variational mechanics and discrete variational mechanics following the work of [64]. Especially, we focus on the Lagrangian and Hamiltonian description of control forces for the established framework of variational mechanics. Definitions and concepts of the variational principle, the Legendre transform, and Noether's theorem are readopted for the forced case in both the continuous as well as the discrete setting. In Sections 2.1 and 2.2 we combine concepts from optimal control and discrete variational mechanics to build up a setting for the optimal control of a continuous and a discrete mechanical system, respectively. Section 1.3 describes the correspondence between the continuous and the discrete Lagrangian system as basis for a comparison between the continuous and the discrete optimal control problems in Section 2.3: We link both frameworks viewing the discrete problem as an approximation of the continuous one. The application of discrete variational principles for a discrete description of the dynamical system leads to structure-preserving time-stepping equations. Here, the special benefits of variational integrators are handed down to the optimal control context. These time-stepping equations serve as equality constraints for the resulting finite dimensional nonlinear optimization problem, therefore the described procedure can be categorized as a direct solution method. Furthermore, we show the equivalence of the discrete Lagrangian optimal control problems to those resulting from Runge-Kutta discretizations of the corresponding Hamiltonian system. This equivalence allows us to construct and compare the adjoint systems of the continuous and the discrete Lagrangian optimal control problem. In this way, one of our main results is related to the order of approximation of the adjoint system of the discrete optimal control problem to that of the continuous one. With the help of this approximation result, we show that the solution of the discrete Lagrangian optimal control system converges to the continuous solution of the original optimal control problem. The proof strategy is based on existing convergence results of optimal control problems discretized via Runge-Kutta methods [21,28]. Section 3.1 gives a detailed description of implementation issues of our method. Furthermore, in Section 3.2 we numerically verify the preservation and convergence properties of DMOC and the benefits of using DMOC compared to other standard methods to the solution of optimal control problems.

\section{Mechanichl systems With Forcing And CONTROL}

\subsection{Variational mechanics}

Our aim is to optimally control Lagrangian and Hamiltonian systems. For the description of their dynamics, we introduce a variational framework including external forcing resulting from dissipation, friction, loading and in particular control forces. To this end, we extend the notions in [64] to Lagrangian control forces.

Forced Lagrangian systems. Consider an $n$-dimensional configuration manifold $Q$ with local coordinates $q=\left(q^{1}, \ldots, q^{n}\right)$, the associated state space given by the tangent bundle $T Q$ and a $C^{k}$ Lagrangian $L: T Q \rightarrow \mathbb{R}$, $k \geq 2$. Given a time interval $[0, \mathrm{~T}]$, we consider curves $q$ in the path space $C^{1,1}([0, \mathrm{~T}], Q)^{4}$ and the action map $\mathfrak{G}: C^{1,1}([0, \mathrm{~T}], Q) \rightarrow \mathbb{R}$

$$
\mathfrak{G}(q)=\int_{0}^{\mathrm{T}} L(q(t), \dot{q}(t)) \mathrm{d} t .
$$

To define control forces for Lagrangian systems, we introduce a control manifold $U \subset \mathbb{R}^{m}$ and define the control path space $L^{\infty}([0, T], U)$ with $u(t) \in U$ also called the control parameter ${ }^{5}$. With this notation we define a Lagrangian control force as a map $f_{L}: T Q \times U \rightarrow T^{*} Q$, which is given in coordinates as $f_{L}$ : $(q, \dot{q}, u) \mapsto\left(q, f_{L}(q, \dot{q}, u)\right)$, where we assume that the control forces can also include configuration and velocity dependent forces resulting e.g. from dissipation and friction. We interpret a Lagrangian control force as a family of Lagrangian forces that are fiber-preserving maps $f_{L}^{u}: T Q \rightarrow T^{*} Q$ over the identity $i d_{Q}{ }^{6}$, i.e. in coordinates $f_{L}^{u}:(q, \dot{q}) \mapsto\left(q, f_{L}^{u}(q, \dot{q})\right)$. Whenever we denote $f_{L}(q, \dot{q}, u)$ as a one-form on $T Q$, we mean the family

\footnotetext{
${ }^{4} C^{1,1}([0, \mathrm{~T}], Q)$ is the space of functions $q:[0, \mathrm{~T}] \rightarrow Q$ which are continuously differentiable on $(0, \mathrm{~T})$ and whose first derivative is Lipschitz continuous on $[0, T]$.

${ }^{5} L^{\infty}$ denotes the space of essentially bounded, measurable functions equipped with the essential supremum norm.

${ }^{6} \mathrm{~A}$ map $\varphi: T^{*} S \rightarrow T^{*} Q$ is called fiber preserving if $f \circ \pi_{Q}=\pi_{S} \circ \varphi^{-1}$ with $\pi_{Q}: T^{*} Q \rightarrow Q, \pi_{S}: T^{*} S \rightarrow S$ the canonical projections and where $f: Q \rightarrow S$ is defined by $f=\varphi^{-1} \mid Q$.
} 
of horizontal one-forms $f_{L}^{u}(q, \dot{q})$ on $T Q$ induced by the family of fiber-preserving maps $f_{L}^{u}$. Given a control path $u \in L^{\infty}([0, T], U)$, the Lagrange-d'Alembert principle seeks curves $q \in C^{1,1}([0, T], Q)$ satisfying

$$
\delta \int_{0}^{\mathrm{T}} L(q(t), \dot{q}(t)) \mathrm{d} t+\int_{0}^{\mathrm{T}} f_{L}(q(t), \dot{q}(t), u(t)) \cdot \delta q(t) \mathrm{d} t=0,
$$

where $\delta$ represents variations vanishing at the endpoints. The second integral in (1.2) is the virtual work acting on the mechanical system via the force $f_{L}$. Integration by parts shows that this is equivalent to the forced Euler-Lagrange equations

$$
\frac{\partial L}{\partial q}(q, \dot{q})-\frac{\mathrm{d}}{\mathrm{d} t}\left(\frac{\partial L}{\partial \dot{q}}(q, \dot{q})\right)+f_{L}(q, \dot{q}, u)=0
$$

These equations implicitly define a family of forced Lagrangian vector fields $X_{L}^{u}: T Q \times[0, T] \rightarrow T(T Q)$ and associated forced Lagrangian flows $F_{L}^{u}: T Q \times[0, T] \rightarrow T Q\left(u \in L^{\infty}([0, T], U)\right.$ fixed).

Remark 1.1. We distinguish between two notations: When we fix $u \in U$ we always consider a family of Lagrangian control forces $f_{L}^{u}$. As soon as we consider evolutions given by differential equations or integrals, instead of fixing only one $u \in U$, we fix an entire curve $u \in L^{\infty}([0, T], U)$, such that for each time $t$ we use the force $f_{L}$ that corresponds to $f_{L}(q(t), \dot{q}(t), u(t))$. In particular, by fixing a control path $u \in L^{\infty}([0, T], U)$ we obtain a non-autonomous system whose evolution is also dependent on the initial time $t_{0}$, such that the flow $F_{L}^{u}$ would be defined on $T Q \times[0, T]^{2}$ rather than on $T Q \times[0, T]$. In the following we will fix the initial time to be $t_{0}=0$ so that we do not need to keep track on the initial time in the notation. This is no restriction since we consider all possible control paths $u \in L^{\infty}([0, \mathrm{~T}], U)$.

The one-form $\Theta_{L}$ on $T Q$ given in coordinates by $\Theta_{L}=\frac{\partial L}{\partial \dot{q}^{i}} \mathbf{d} q^{i}$ is called the Lagrangian one-form, and the Lagrangian symplectic form $\Omega_{L}=\mathbf{d} \Theta_{L}$ is given in coordinates by $\Omega_{L}(q, \dot{q})=\frac{\partial^{2} L}{\partial q^{i} \partial \dot{q}^{j}} \mathbf{d} q^{i} \wedge \mathbf{d} q^{j}+\frac{\partial^{2} L}{\partial \dot{q}^{i} \partial \dot{q}^{j}} \mathbf{d} \dot{q}^{i} \wedge \mathbf{d} q^{j}$. Recall that in the absence of forces, the Lagrangian symplectic form is preserved under the Lagrangian flow [64].

Forced Hamiltonian systems. Consider an $n$-dimensional configuration manifold $Q$, and define the phase space to be the cotangent bundle $T^{*} Q$. The Hamiltonian is a function $H: T^{*} Q \rightarrow \mathbb{R}$. We will take local coordinates on $T^{*} Q$ to be $(q, p)$ with $q=\left(q^{1}, \ldots, q^{n}\right)$ and $p=\left(p_{1}, \ldots, p_{n}\right)$. Define the canonical one-form $\Theta$ on $T^{*} Q$ by $\Theta\left(p_{q}\right) \cdot u_{p_{q}}=\left\langle p_{q}, T_{\pi_{Q}} \cdot u_{p_{q}}\right\rangle$, where $p_{q} \in T^{*} Q, u_{p_{q}} \in T_{p_{q}}\left(T^{*} Q\right), \pi_{Q}: T^{*} Q \rightarrow Q$ is the canonical projection, $T_{\pi_{Q}}: T\left(T^{*} Q\right) \rightarrow T Q$ is the tangent map of $\pi_{Q}$ and $\langle\cdot, \cdot\rangle$ denotes the natural pairing between vectors and covectors. In coordinates, we have $\Theta(q, p)=p_{i} \mathbf{d} q^{i}$. The canonical two-form $\Omega$ on $T^{*} Q$ is defined to be $\Omega=-\mathbf{d} \Theta$, which has the coordinate expression $\Omega(q, p)=\mathbf{d} q^{i} \wedge \mathbf{d} p_{i}$. A Hamiltonian control force is a map $f_{H}: T^{*} Q \times U \rightarrow T^{*} Q$ identified by a family of fiber preserving maps $f_{H}^{u}: T^{*} Q \rightarrow T^{*} Q$ over the identity. Given such a control force, we define the corresponding family of horizontal one-forms $f_{H}^{\prime}$ on $T^{*} Q$ by $\left(f_{H}^{u}\right)^{\prime}\left(p_{q}\right) \cdot w_{p_{q}}=\left\langle f_{H}^{u}\left(p_{q}\right), T_{\pi_{Q}} \cdot w_{p_{q}}\right\rangle$. This expression is reminiscent of the canonical one-form $\Theta$ on $T^{*} Q$, and in coordinates it reads $\left(f_{H}^{u}\right)^{\prime}(q, p) \cdot(\delta q, \delta p)=f_{H}^{u}(q, p) \cdot \delta q$. For a given curve $u \in L^{\infty}([0, \mathrm{~T}], U)$, the forced Hamiltonian vector field $X_{H}^{u}$ is now defined by the equation $\mathbf{i}_{X_{H}^{u}} \Omega=\mathbf{d} H-\left(f_{H}^{u}\right)^{\prime}$ and in coordinates this gives the well-known forced Hamilton's equations

$$
X_{q}^{u}(q, p)=\frac{\partial H}{\partial p}(q, p), \quad X_{p}^{u}(q, p)=-\frac{\partial H}{\partial q}(q, p)+f_{H}^{u}(q, p),
$$

which are the standard Hamilton's equations in coordinates with the forcing term added to the momentum equation. This defines the forced Hamiltonian flow $F_{H}^{u}: T^{*} Q \times[0, \mathrm{~T}] \rightarrow T^{*} Q$ of the forced Hamiltonian vector field $X_{H}^{u}=\left(X_{q}^{u}, X_{p}^{u}\right)\left(u \in L^{\infty}([0, \mathrm{~T}], U)\right.$ fixed).

The Legendre transform with forces. Given a Lagrangian $L$, we can take the standard Legendre transform $\mathbb{F} L: T Q \rightarrow T^{*} Q$ defined by

$$
\mathbb{F} L\left(v_{q}\right) \cdot w_{q}=\left.\frac{\mathrm{d}}{\mathrm{d} \epsilon}\right|_{\epsilon=0} L\left(v_{q}+\epsilon w_{q}\right),
$$


where $v_{q}, w_{q} \in T_{q} Q$, and which has coordinate form $\mathbb{F} L:(q, \dot{q}) \mapsto(q, p)=(q, \partial L / \partial \dot{q}(q, \dot{q}))$, and relate Hamiltonian and Lagrangian control forces by $f_{L}^{u}=f_{H}^{u} \circ \mathbb{F} L$. If we also have a Hamiltonian $H$ related to $L$ by the Legendre transform as $H(q, p)=\mathbb{F} L(q, \dot{q}) \cdot \dot{q}-L(q, \dot{q})$, then the forced Euler-Lagrange equations and the forced Hamilton's equations are equivalent. That is, if $X_{L}^{u}$ and $X_{H}^{u}$ are the forced Lagrangian and Hamiltonian vector fields, respectively, then $(\mathbb{F} L)^{*}\left(X_{H}^{u}\right)=X_{L}^{u}, c f$. [64].

Noether's theorem with forcing. A key property of Lagrangian flows is their behavior with respect to group actions. Assume a Lie group $G$ with Lie algebra $\mathfrak{g}$ acts on $Q$ by the (left or right) action $\phi: G \times Q \rightarrow Q$. Consider the tangent lift of this action to $\phi^{T Q}: G \times T Q$ given by $\phi_{g}^{T Q}\left(v_{q}\right)=T\left(\phi_{g}\right) \cdot v_{q}$. For $\xi \in \mathfrak{g}$ the infinitesimal generators $\xi_{Q}: Q \rightarrow T Q$ and $\xi_{T Q}: T Q \rightarrow T(T Q)$ are defined by $\xi_{Q}(q)=\frac{\mathrm{d}}{\mathrm{d} q}\left(\phi_{g}(q)\right) \cdot \xi$ and $\xi_{T Q}\left(v_{q}\right)=\frac{\mathrm{d}}{\mathrm{d} g}\left(\phi_{g}^{T Q}\left(v_{q}\right)\right) \cdot \xi$, and the Lagrangian momentum map $\mathbf{J}_{L}: T Q \rightarrow \mathfrak{g}^{*}$ is defined to be $\mathbf{J}_{L}\left(v_{q}\right) \cdot \xi=$ $\Theta_{L} \cdot \xi_{T Q}\left(v_{q}\right)$. If the Lagrangian is invariant under the lift of the action, that is we have $L \circ \phi_{g}^{T Q}=L$ for all $g \in G$ (we also say, the group action is a symmetry of the Lagrangian), the Lagrangian momentum map is preserved of the Lagrangian flow in the absence of external forces. We now consider the effect of forcing on the evolution of momentum maps that arise from symmetries of the Lagrangian. In [64] it is shown that the evolution of the momentum map from time 0 to time $\mathrm{T}$ is given by the relation

$$
\left[\left(\mathbf{J}_{L} \circ\left(F_{L}^{u}\right)^{T}\right)(q(0), \dot{q}(0))-\mathbf{J}_{L}(q(0), \dot{q}(0))\right] \cdot \xi=\int_{0}^{\mathrm{T}} f_{L}^{u}(q(t), \dot{q}(t)) \cdot \xi_{Q}(q(t)) \mathrm{d} t .
$$

Equation (1.5) shows, that forcing will generally alter the momentum map. However, in the special case that the forcing is orthogonal to the group action, the above relation shows that Noether's theorem will still hold.

Theorem 1.2 (forced Noether's theorem). Consider a Lagrangian system $L: T Q \rightarrow \mathbb{R}$ with control forcing $f_{L}: T Q \times U \rightarrow T^{*} Q$ such that the Lagrangian is invariant under the lift of the (left or right) action $\phi: G \times Q \rightarrow Q$ and $\left\langle f_{L}^{u}(q, \dot{q}), \xi_{Q}(q)\right\rangle=0$ for all $(q, \dot{q}) \in T Q, u \in U$ and all $\xi \in \mathfrak{g}$. Then the Lagrangian momentum map $\mathbf{J}_{L}: T Q \rightarrow \mathfrak{g}^{*}$ will be preserved by the flow, such that $\mathbf{J}_{L} \circ\left(F_{L}^{u}\right)^{t}=\mathbf{J}_{L}$ for all $t$.

\subsection{Discrete mechanics}

The discrete Lagrangian. Again we consider a configuration manifold $Q$, and define the ("discrete") state space to be $Q \times Q$. Rather than considering a configuration $q$ and velocity $\dot{q}$ (or momentum $p$ ), we now consider two configurations $q_{0}$ and $q_{1}$, which should be thought of as two points on a curve $q$ which are a time step $h>0$ apart, i.e. $q_{0} \approx q(0)$ and $q_{1} \approx q(h)$. The manifold $Q \times Q$ is locally isomorphic to $T Q$ and thus contains the same amount of information. A discrete Lagrangian is a function $L_{d}: Q \times Q \rightarrow \mathbb{R}$, which we think of as approximating the action integral along the exact solution curve segment $q$ between $q_{0}$ and $q_{1}$ : $L_{d}\left(q_{0}, q_{1}\right) \approx \int_{0}^{h} L(q(t), \dot{q}(t)) \mathrm{d} t$. We consider the grid $\left\{t_{k}=k h \mid k=0, \ldots, N\right\}, N h=T$, and define the discrete path space $\mathcal{P}_{d}(Q)=\left\{q_{d}:\left\{t_{k}\right\}_{k=0}^{N} \rightarrow Q\right\}$. We will identify a discrete trajectory $q_{d} \in \mathcal{P}_{d}(Q)$ with its image $q_{d}=\left\{q_{k}\right\}_{k=0}^{N}$, where $q_{k}=q_{d}\left(t_{k}\right)$. The discrete action map $\mathfrak{G}_{d}: \mathcal{P}_{d}(Q) \rightarrow \mathbb{R}$ along this sequence is calculated by summing the discrete Lagrangian on each adjacent pair and defined by $\mathfrak{G}_{d}\left(q_{d}\right)=\sum_{k=0}^{N-1} L_{d}\left(q_{k}, q_{k+1}\right)$. As the discrete path space $\mathcal{P}_{d}$ is isomorphic to $Q \times \ldots \times Q(N+1$ copies $)$, it can be given a smooth product manifold structure. The discrete action $\mathfrak{G}_{d}$ inherits the smoothness of the discrete Lagrangian $L_{d}$. The tangent space $T_{q_{d}} \mathcal{P}_{d}(Q)$ to $\mathcal{P}_{d}(Q)$ at $q_{d}$ is the set of maps $v_{q_{d}}:\left\{t_{k}\right\}_{k=0}^{N} \rightarrow T Q$ such that $\tau_{q} \circ v_{q_{d}}=q_{d}$, which we will denote by $v_{q_{d}}=\left\{\left(q_{k}, v_{k}\right)\right\}_{k=0}^{N}$. To complete the discrete setting for forced mechanical systems, we present a discrete formulation of the control forces introduced in the previous section. Since the control path $u:[0, T] \rightarrow U$ has no geometric interpretation, we have to find an appropriate discrete formulation to identify a discrete structure for the Lagrangian control force.

Discrete Lagrangian control forces. Analogous to the replacement of the path space by a discrete path space, we replace the control path space by a discrete one. To this end we consider a refined grid $\Delta \tilde{t}$, generated via a set of control points $0 \leq c_{1}<\ldots<c_{s} \leq 1$ as $\Delta \tilde{t}=\left\{t_{k \ell}=t_{k}+c_{\ell} h \mid k=0, \ldots, N-1, \ell=1, \ldots, s\right\}$. With this notation the discrete control path space is defined to be $\mathcal{P}_{d}(U)=\left\{u_{d}: \Delta \tilde{t} \rightarrow U\right\}$. We define the intermediate 
control samples $u_{k}$ on $\left[t_{k}, t_{k+1}\right]$ as $u_{k}=\left(u_{k 1}, \ldots, u_{k s}\right) \in U^{s}$ to be the values of the control parameters guiding the system from $q_{k}=q_{d}\left(t_{k}\right)$ to $q_{k+1}=q_{d}\left(t_{k+1}\right)$, where $u_{k l}=u_{d}\left(t_{k l}\right)$ for $l \in\{1, \ldots, s\}$. With this definition of the discrete control path space, we take two discrete Lagrangian control forces $f_{d}^{+}, f_{d}^{-}: Q \times Q \times U^{s} \rightarrow T^{*} Q$, given in coordinates as

$$
f_{d}^{+}\left(q_{k}, q_{k+1}, u_{k}\right)=\left(q_{k+1}, f_{d}^{+}\left(q_{k}, q_{k+1}, u_{k}\right)\right), \quad f_{d}^{-}\left(q_{k}, q_{k+1}, u_{k}\right)=\left(q_{k}, f_{d}^{-}\left(q_{k}, q_{k+1}, u_{k}\right)\right),
$$

also called left and right discrete forces ${ }^{7}$. Analogously to the continuous case, we interpret the two discrete Lagrangian control forces as two families of discrete fiber-preserving Lagrangian forces $f_{d}^{u_{k}, \pm}: Q \times Q \rightarrow T^{*} Q$ in the sense that $\pi_{Q} \circ f_{d}^{u_{k}, \pm}=\pi_{Q}^{ \pm}$with fixed $u_{k} \in U^{s}$ and with the projection operators $\pi_{Q}^{+}: Q \times Q \rightarrow Q$, $\left(q_{k}, q_{k+1}\right) \mapsto q_{k+1}$ and $\pi_{Q}^{-}: Q \times Q \rightarrow Q,\left(q_{k}, q_{k+1}\right) \mapsto q_{k}$. We combine the two discrete control forces to give a single one-form $f_{d}^{u_{k}}: Q \times Q \rightarrow T^{*}(Q \times Q)$ defined by

$$
f_{d}^{u_{k}}\left(q_{k}, q_{k+1}\right) \cdot\left(\delta q_{k}, \delta q_{k+1}\right)=f_{d}^{u_{k},+}\left(q_{k}, q_{k+1}\right) \cdot \delta q_{k+1}+f_{d}^{u_{k},-}\left(q_{k}, q_{k+1}\right) \cdot \delta q_{k},
$$

where $f_{d}\left(q_{k}, q_{k+1}, u_{k}\right)$ denotes the family of all one-forms $f_{d}^{u_{k}}\left(q_{k}, q_{k+1}\right)$ with fixed $u_{k} \in U^{s}$. To simplify the notation we denote the left and right discrete forces by $f_{k}^{ \pm}:=f_{d}^{ \pm}\left(q_{k}, q_{k+1}, u_{k}\right)$, respectively, and the pair consisting of both by $f_{k}:=f_{d}\left(q_{k}, q_{k+1}, u_{k}\right)$. We interpret the left discrete force $f_{k-1}^{+}$(and right discrete force $f_{k}^{-}$, respectively) as the force resulting from the continuous control force acting during the time span $\left[t_{k-1}, t_{k}\right]$ (during the time span $\left[t_{k}, t_{k+1}\right]$, respectively) on the configuration node $q_{k}$.

The discrete Lagrange-d'Alembert principle. As with discrete Lagrangians, the discrete control forces also depend on the time step $h$, which is important when relating discrete and continuous mechanics. Given such forces, we modify the discrete Hamilton's principle, following [39], to the discrete Lagrange-d'Alembert principle, which seeks discrete curves $\left\{q_{k}\right\}_{k=0}^{N}$ that satisfy

$$
\delta \sum_{k=0}^{N-1} L_{d}\left(q_{k}, q_{k+1}\right)+\sum_{k=0}^{N-1}\left[f_{d}^{-}\left(q_{k}, q_{k+1}, u_{k}\right) \cdot \delta q_{k}+f_{d}^{+}\left(q_{k}, q_{k+1}, u_{k}\right) \cdot \delta q_{k+1}\right]=0
$$

for all variations $\left\{\delta q_{k}\right\}_{k=0}^{N}$ vanishing at the endpoints. This is equivalent to the forced discrete Euler-Lagrange equations

$$
D_{2} L_{d}\left(q_{k-1}, q_{k}\right)+D_{1} L_{d}\left(q_{k}, q_{k+1}\right)+f_{d}^{+}\left(q_{k-1}, q_{k}, u_{k-1}\right)+f_{d}^{-}\left(q_{k}, q_{k+1}, u_{k}\right)=0, \quad k=1, \ldots, N-1 .
$$

These equations implicitly define the forced discrete Lagrangian map $F_{L_{d}}^{u_{k-1}, u_{k}}: Q \times Q \rightarrow Q \times Q$ for fixed controls $u_{k-1}, u_{k} \in U^{s}$, mapping $\left(q_{k-1}, q_{k}\right)$ to $\left(q_{k}, q_{k+1}\right)$. The discrete Lagrangian one-forms $\Theta_{L_{d}}^{+}$and $\Theta_{L_{d}}^{-}$are in coordinates $\Theta_{L_{d}}^{+}\left(q_{0}, q_{1}\right)=D_{2} L_{d}\left(q_{0}, q_{1}\right) \mathbf{d} q_{1}$ and $\Theta_{L_{d}}^{-}\left(q_{0}, q_{1}\right)=-D_{1} L_{d}\left(q_{0}, q_{1}\right) \mathbf{d} q_{0}$. In the absence of external forces, the discrete Lagrangian maps inherit the properties of symplectic preservation from the continuous Lagrangian flows. That means the discrete Lagrangian symplectic form $\Omega_{L_{d}}=\mathbf{d} \Theta_{L_{d}}^{+}=\mathbf{d} \Theta_{L_{d}}^{-}$(see [64] for the coordinate expression) is preserved under the discrete Lagrangian map as $\left(F_{L_{d}}\right)^{*}\left(\Omega_{L_{d}}\right)=\Omega_{L_{d}}$, if no external forcing is present.

The discrete Legendre transforms with forces. Although in the continuous case we used the standard Legendre transform for systems with forcing, in the discrete case it is necessary to take the forced discrete Legendre transforms

$$
\begin{aligned}
& \mathbb{F}^{f+} L_{d}:\left(q_{0}, q_{1}, u_{0}\right) \mapsto\left(q_{1}, p_{1}\right)=\left(q_{1}, D_{2} L_{d}\left(q_{0}, q_{1}\right)+f_{d}^{+}\left(q_{0}, q_{1}, u_{0}\right)\right), \\
& \mathbb{F}^{f-} L_{d}:\left(q_{0}, q_{1}, u_{0}\right) \mapsto\left(q_{0}, p_{0}\right)=\left(q_{0},-D_{1} L_{d}\left(q_{0}, q_{1}\right)-f_{d}^{-}\left(q_{0}, q_{1}, u_{0}\right)\right)
\end{aligned}
$$

\footnotetext{
${ }^{7}$ Observe that the discrete control force is now dependent on the discrete control path.
} 
Again, we denote with $\mathbb{F}^{f \pm} L_{d}^{u_{0}}$ the forced discrete Legendre transforms for fixed controls $u_{0} \in U^{s}$. Using these definitions and the forced discrete Euler-Lagrange equations (1.9), we can see that the corresponding forced discrete Hamiltonian map $\tilde{F}_{L_{d}}^{u_{0}}=\mathbb{F}^{f \pm} L_{d}^{u_{1}} \circ F_{L_{d}}^{u_{0}, u_{1}} \circ\left(\mathbb{F}^{f \pm} L_{d}^{u_{0}}\right)^{-1}$ is given by the map $\tilde{F}_{L_{d}}^{u_{0}}:\left(q_{0}, p_{0}\right) \mapsto\left(q_{1}, p_{1}\right)$, where

$$
p_{0}=-D_{1} L_{d}\left(q_{0}, q_{1}\right)-f_{d}^{u_{0},-}\left(q_{0}, q_{1}\right), \quad p_{1}=D_{2} L_{d}\left(q_{0}, q_{1}\right)+f_{d}^{u_{0},+}\left(q_{0}, q_{1}\right),
$$

which is the same as the standard discrete Hamiltonian map with the discrete forces added.

As in [64] one can show that the following two definitions of the forced discrete Hamiltonian map

$$
\tilde{F}_{L_{d}}^{u_{0}}=\mathbb{F}^{f \pm} L_{d}^{u_{1}} \circ F_{L_{d}}^{u_{0}, u_{1}} \circ\left(\mathbb{F}^{f \pm} L_{d}^{u_{0}}\right)^{-1}, \quad \tilde{F}_{L_{d}}^{u_{0}}=\mathbb{F}^{f+} L_{d}^{u_{0}} \circ\left(\mathbb{F}^{f-} L_{d}^{u_{0}}\right)^{-1}
$$

are equivalent with coordinate expression (1.11). Thus from the second expression in (1.12) it becomes clear, that the forced discrete Hamiltonian map that maps $\left(q_{0}, p_{0}\right)$ to $\left(q_{1}, p_{1}\right)$ depends on $u_{0}$ only.

The discrete Noether theorem with forcing. As in the unforced case, we can formulate a discrete version of the forced Noether's theorem (for the derivation see for example [64]). To this end, the discrete momentum map in presence of forcing is defined as

$$
\mathbf{J}_{L_{d}}^{f+}\left(q_{0}, q_{1}\right) \cdot \xi=\left\langle\mathbb{F}^{f+} L_{d}^{u_{0}}\left(q_{0}, q_{1}\right), \xi_{Q}\left(q_{1}\right)\right\rangle, \quad \mathbf{J}_{L_{d}}^{f-}\left(q_{0}, q_{1}\right) \cdot \xi=\left\langle\mathbb{F}^{f-} L_{d}^{u_{0}}\left(q_{0}, q_{1}\right), \xi_{Q}\left(q_{0}\right)\right\rangle .
$$

The evolution of the discrete momentum map is described by

$$
\left[\mathbf{J}_{L_{d}}^{f+} \circ\left(F_{L_{d}}^{u_{d}}\right)^{N-1}-\mathbf{J}_{L_{d}}^{f-}\right]\left(q_{0}, q_{1}\right) \cdot \xi=\sum_{k=0}^{N-1} f_{d}^{u_{k}}\left(q_{k}, q_{k+1}\right) \cdot \xi_{Q \times Q}\left(q_{k}, q_{k+1}\right) .
$$

Again, in the case that the forcing is orthogonal to the group action we have the unique momentum map $\mathbf{J}_{L_{d}}^{f}: Q \times Q \rightarrow \mathfrak{g}^{*}$ and it holds:

Theorem 1.3 (forced discrete Noether's theorem). Consider a discrete Lagrangian system $L_{d}: Q \times Q \rightarrow \mathbb{R}$ with discrete control forces $f_{d}^{+}, f_{d}^{-}: Q \times Q \times U^{s} \rightarrow T^{*} Q$ such that the discrete Lagrangian is invariant under the lift of the (left or right) action $\phi: G \times Q \rightarrow Q$ and $\left\langle f_{d}^{u_{k}}, \xi_{Q \times Q}\right\rangle=0$ for all $\xi \in \mathfrak{g}$ and $u_{k} \in U^{s}, k \in\{0, \ldots, N-1\}$. Then the discrete Lagrangian momentum map $\mathbf{J}_{L_{d}}^{f}: Q \times Q \rightarrow \mathfrak{g}^{*}$ will be preserved by the discrete Lagrangian evolution map, such that $\mathbf{J}_{L_{d}}^{f} \circ F_{L_{d}}^{u_{k}, u_{k+1}}=\mathbf{J}_{L_{d}}^{f}$.

\subsection{The discrete $v s$. the continuous Lagrangian systems}

In this section, we relate the continuous and the discrete Lagrangian system. First, along the lines of [64], we define expressions for the discrete mechanical objects that exactly reflect the continuous ones. Based on the exact discrete expressions, we determine the order of consistency concerning the difference between the continuous and the discrete mechanical system.

Exact discrete Lagrangian and forcing. Given a regular Lagrangian $L: T Q \rightarrow \mathbb{R}$ and a Lagrangian control force $f_{L}: T Q \times U \rightarrow T^{*} Q$, we define the exact discrete Lagrangian $L_{d}^{E}: Q \times Q \times \mathbb{R} \rightarrow \mathbb{R}$ and the exact discrete control forces $f_{d}^{E+}, f_{d}^{E-}: Q \times Q \times L^{\infty}([0, h], U) \times \mathbb{R} \rightarrow T^{*} Q$ to be

$$
\begin{aligned}
L_{d}^{E}\left(q_{0}, q_{1}, h\right)=\int_{0}^{h} L(q(t), \dot{q}(t)) \mathrm{d} t, \quad & f_{d}^{E+}\left(q_{0}, q_{1}, \mathrm{u}_{0}, h\right)=\int_{0}^{h} f_{L}(q(t), \dot{q}(t), u(t)) \cdot \frac{\partial q(t)}{\partial q_{1}} \mathrm{~d} t \\
f_{d}^{E-}\left(q_{0}, q_{1}, \mathrm{u}_{0}, h\right) & =\int_{0}^{h} f_{L}(q(t), \dot{q}(t), u(t)) \cdot \frac{\partial q(t)}{\partial q_{0}} \mathrm{~d} t
\end{aligned}
$$

with $\mathrm{u}_{k} \in L^{\infty}([k h,(k+1) h], U)$ and where $q:[0, h] \rightarrow Q$ is the solution of the forced Euler-Lagrange equations (1.3) with control function $u:[0, h] \rightarrow U$ for $L$ and $f_{L}$ satisfying the boundary conditions $q(0)=q_{0}$ 


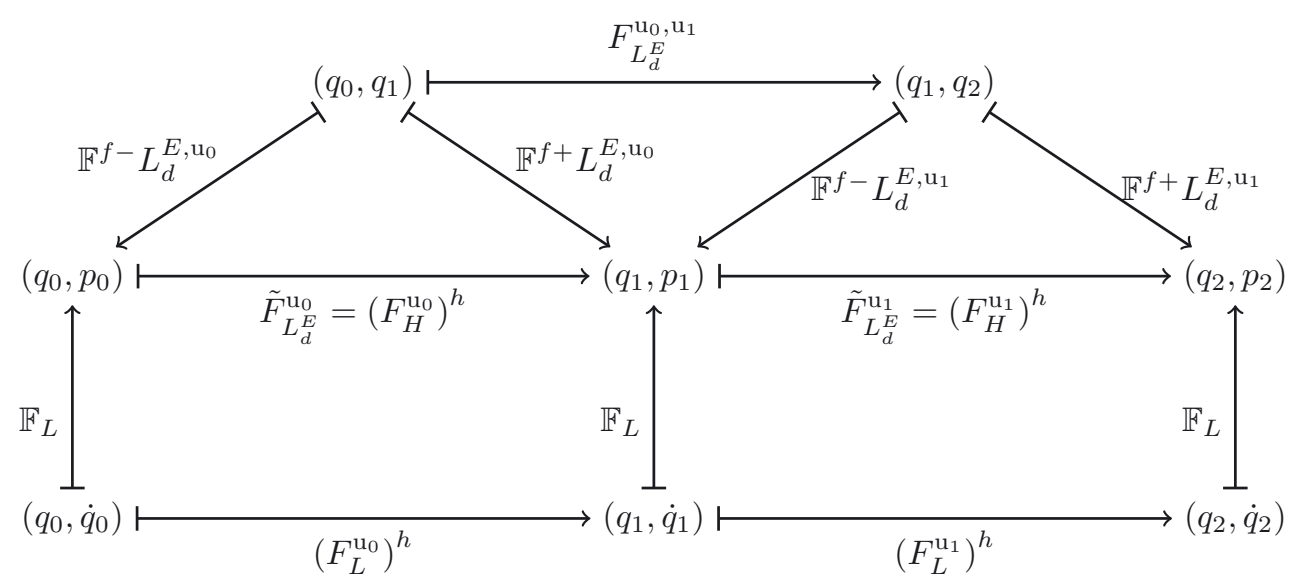

Figure 1. Correspondence between the exact discrete Lagrangian and forces and the continuous forced Hamiltonian flow.

and $q(h)=q_{1}$. Observe, that the exact discrete control forces depend on an entire control path in contrast to the continuous control forces. Consequently, the exact forced discrete Legendre transforms are given by

$$
\begin{aligned}
& \mathbb{F}^{f+} L_{d}^{E}\left(q_{0}, q_{1}, \mathrm{u}_{0}, h\right)=\left(q_{1}, D_{2} L_{d}^{E}\left(q_{0}, q_{1}, h\right)+f_{d}^{E+}\left(q_{0}, q_{1}, \mathrm{u}_{0}, h\right)\right), \\
& \mathbb{F}^{f-} L_{d}^{E}\left(q_{0}, q_{1}, \mathrm{u}_{0}, h\right)=\left(q_{0},-D_{1} L_{d}^{E}\left(q_{0}, q_{1}, h\right)-f_{d}^{E-}\left(q_{0}, q_{1}, \mathrm{u}_{0}, h\right)\right) .
\end{aligned}
$$

As in Section $1.2 \mathbb{F}^{f \pm} L_{d}^{E, \mathrm{u}_{k}}\left(q_{k}, q_{k+1}\right)$ and $f_{d}^{E, \mathrm{u}_{k}, \pm}\left(q_{k}, q_{k+1}\right)$ denote the exact discrete forces and the exact forced discrete Legendre transforms for a fixed $\mathrm{u}_{k} \in L^{\infty}([k h,(k+1) h], U)$. As in [64] (Lem. 1.6.2) by taking the presence of control forces into account we obtain that a regular Lagrangian $L$ and the corresponding exact discrete Lagrangian $L_{d}^{E}$ have Legendre transforms related by

$$
\mathbb{F}^{f+} L_{d}^{E}\left(q_{0}, q_{1}, \mathrm{u}_{0}, h\right)=\mathbb{F} L\left(q_{0,1}(h), \dot{q}_{0,1}(h)\right), \quad \mathbb{F}^{f-} L_{d}^{E}\left(q_{0}, q_{1}, \mathrm{u}_{0}, h\right)=\mathbb{F} L\left(q_{0,1}(0), \dot{q}_{0,1}(0)\right),
$$

for sufficiently small $h$ and close $q_{0}, q_{1} \in Q$. Here $q_{0,1}$ denotes the solution of the corresponding Euler-Lagrange equations with $q(0)=q_{0}, q(h)=q_{1}$. This also proves that exact discrete Lagrangians are automatically regular.

Combining this result with the relations (1.12) gives the commutative diagram shown in Figure 1 for the exact discrete Lagrangian and forces. The diagram also clarifies the following observation, that was already proved in [64] (Thm. 1.6.3) for unforced systems and can now be established for the forced case as well: consider the pushforward of both, the continuous Lagrangian and forces and their exact discrete Lagrangian and discrete forces to $T^{*} Q$, yielding a forced Hamiltonian system with Hamiltonian $H$ and a forced discrete Hamiltonian map $\tilde{F}_{L_{d}^{E}}^{u_{k}}$, respectively. Then, for a sufficiently small time step $h \in \mathbb{R}$, the forced Hamiltonian flow map equals the pushforward discrete Lagrangian map: $\left(F_{H}^{\mathrm{u}_{0}}\right)^{h}=\tilde{F}_{L_{d}^{E}}^{\mathrm{u}_{0}}$.

Order of consistency. In the previous paragraph we observed that the exact discrete Lagrangian and forces generate a forced discrete Hamiltonian map that exactly equals the forced Hamiltonian flow of the continuous system. Since we are interested in using discrete mechanics to reformulate optimal control problems, we generally do not assume that $L_{d}$ and $L$ or $H$ are related by (1.14). Moreover, the exact discrete Lagrangian and exact discrete forces are generally not computable. In this section we determine the error we obtain by using discrete approximations for the Lagrangian and the control forces and make use of the concept of variational error analysis (introduced in [64]). In this context, it is considered how closely a discrete Lagrangian matches the exact discrete Lagrangian given by the action. Thus, a given discrete Lagrangian $L_{d}$ is of order $r$, if for a fixed 
curve $u \in L^{\infty}([0, T], U)$ there exist an open subset $V_{v} \subset T Q$ with compact closure and constants $C_{v}>0$ and $h_{v}>0$ such that

$$
\left|L_{d}(q(0), q(h), h)-L_{d}^{E}(q(0), q(h), h)\right| \leq C_{v} h^{r+1}
$$

for all solutions $q(t)$ of the forced Euler-Lagrange equations with initial condition $\left(q^{0}, \dot{q}^{0}\right) \in V_{v}$ and for all $h \leq h_{v}$, were we assume $Q$ to be a normed vector space equipped with the norm $\|\cdot\|$. For forced systems, we additionally take into account how closely the discrete forces match the exact discrete forces: Analogously, we define that a given discrete force $f_{d}^{u_{0}, \pm}$ and the discrete Legendre transforms $\mathbb{F}^{+} L_{d}^{u_{0}}$ and $\mathbb{F}^{-} L_{d}^{u_{0}}$ of a discrete Lagrangian $L_{d}$ are of order $r$, if we have $\left\|f_{d}^{u_{0}, \pm}(q(0), q(h), h)-f_{d}^{E, \mathrm{u}_{0}, \pm}(q(0), q(h), h)\right\| \leq C_{w} h^{r+1}$ and $\left\|\mathbb{F}^{ \pm} L_{d}^{u_{0}}(q(0), q(h), h)-\mathbb{F}^{ \pm} L_{d}^{E, u_{0}}(q(0), q(h), h)\right\| \leq C_{f} h^{r+1}$ for existing open subsets $V_{w}, V_{f} \subset T Q$ with compact closure and constants $C_{w}, C_{f}>0$ and $h_{w}, h_{f}>0$ as above, respectively, and with fixed $u_{0} \in U^{s}$ and $\mathrm{u}_{0} \in L^{\infty}([0, h], U)$.

To give a relationship between the orders of a discrete Lagrangian, discrete forces, the forced discrete Legendre transforms, and their forced discrete Hamiltonian maps, we define that $L_{d}^{1}$ is equivalent to $L_{d}^{2}$ if their discrete Hamiltonian maps are equal. For the forced case, we say analogously, that for fixed $u_{k} \in U^{s}$ the discrete pair $\left(L_{d}^{1}, f_{d}^{u_{k}, 1}\right)$ is equivalent to the discrete pair $\left(L_{d}^{2}, f_{d}^{u_{k}, 2}\right)$ if their forced discrete Hamiltonian maps are equal, such that $\tilde{F}_{L_{d}^{1}}^{u_{k}}=\tilde{F}_{L_{d}^{2}}^{u_{k}}$. With $\tilde{F}_{L_{d}^{1}}^{u_{k}}=\mathbb{F}^{f+} L_{d}^{u_{k}, 1} \circ\left(\mathbb{F}^{f-} L_{d}^{u_{k}, 1}\right)^{-1}$, it follows that if $\left(L_{d}^{1}, f_{d}^{u_{k}, 1}\right)$ and $\left(L_{d}^{2}, f_{d}^{u_{k}, 2}\right)$ are equivalent, then their forced discrete Legendre transforms are equal. Thus, equivalent pairs of discrete Lagrangians and control forces generate the same integrators. As in [64] (Thm. 2.3.1) and [72] we get the following equivalent statements:

(i) the forced discrete Hamiltonian map for $\left(L_{d}, f_{d}^{u_{k}, \pm}\right)$ is of order $r$;

(ii) the forced discrete Legendre transforms of $\left(L_{d}, f_{d}^{u_{k}, \pm}\right)$ are of order $r$;

(iii) $\left(L_{d}, f_{d}^{u_{k}, \pm}\right)$ is equivalent to a pair of discrete Lagrangian and discrete forces, both of order $r$.

Note that, given a discrete Lagrangian and discrete forces, their order can be calculated by expanding the expressions for $L_{d}(q(0), q(h), h)$ and $f_{d}^{u_{k}, \pm}$ in a Taylor series in $h$ and comparing these to the same expansions for the exact Lagrangian and the exact forces, respectively. If the series agree up to $r$ terms, then the discrete objects are of order $r$.

\section{Optimal CONTROL OF A MEChANICAL SYSTEM}

\subsection{The continuous setting}

On the configuration space $Q$ we consider a mechanical system described by a regular Lagrangian $L: T Q \rightarrow \mathbb{R}$. Additionally, assume that a Lagrangian control force acts on the system and is defined by a map $f_{L}: T Q \times U \rightarrow$ $T^{*} Q$ with $f_{L}:(q, \dot{q}, u) \mapsto\left(q, f_{L}(q, \dot{q}, u)\right)$ and $u:[0, T] \rightarrow U$, the time-dependent control parameter. Note that the Lagrangian control force may include both dissipative forces within the mechanical system and external control forces resulting from actuators steering the system.

The Lagrangian optimal control problem. We now consider the following optimal control problem: During the time interval $[0, T]$, the mechanical system described by the Lagrangian $L$ is to be moved on a curve $q$ from an initial state $(q(0), \dot{q}(0))=\left(q^{0}, \dot{q}^{0}\right) \in T Q$ to a final state. The motion is influenced via a Lagrangian control force $f_{L}$ with control parameter $u$ such that a given objective functional

$$
J(q, u)=\int_{0}^{\mathrm{T}} C(q(t), \dot{q}(t), u(t)) \mathrm{d} t+\Phi(q(\mathrm{~T}), \dot{q}(\mathrm{~T}))
$$

is minimized. Here $C: T Q \times U \rightarrow \mathbb{R}$ and $\Phi: T Q \rightarrow \mathbb{R}$ (Mayer term) are continuously differentiable cost functions. The final state $(q(\mathrm{~T}), \dot{q}(\mathrm{~T}))$ is required to fulfil a constraint $r\left(q(\mathrm{~T}), \dot{q}(\mathrm{~T}), q^{\mathrm{T}}, \dot{q}^{\mathrm{T}}\right)=0$ with $r: T Q \times T Q \rightarrow \mathbb{R}^{n_{r}}$ 
and $\left(q^{\mathrm{T}}, \dot{q}^{\mathrm{T}}\right) \in T Q$ given. The motion of the system is to satisfy the Lagrange-d'Alembert principle, which requires that

$$
\delta \int_{0}^{\mathrm{T}} L(q(t), \dot{q}(t)) \mathrm{d} t+\int_{0}^{\mathrm{T}} f_{L}(q(t), \dot{q}(t), u(t)) \cdot \delta q(t) \mathrm{d} t=0
$$

for all variations $\delta q$ with $\delta q(0)=\delta q(\mathrm{~T})=0$. In many cases, one encounters additional constraints on the states and controls given by $h(q(t), \dot{q}(t), u(t)) \geq 0$ with $h: T Q \times U \rightarrow \mathbb{R}^{n_{h}}$, where $V \geq 0$ for vectors $V \in \mathbb{R}^{n}$ holds componentwise. To summarize, we are faced with the following:

Problem 2.1 (Lagrangian optimal control problem (LOCP)).

$$
\begin{aligned}
\min _{q \in C^{1,1}([0, \mathrm{~T}], Q), u \in L^{\infty}([0, \mathrm{~T}], U)} J(q, u) & \\
\text { subject to } \quad \delta \int_{0}^{\mathrm{T}} L(q(t), \dot{q}(t)) \mathrm{d} t+\int_{0}^{\mathrm{T}} f_{L}(q(t), \dot{q}(t), u(t)) \cdot \delta q(t) \mathrm{d} t & =0, \\
(q(0), \dot{q}(0)) & =\left(q^{0}, \dot{q}^{0}\right), \\
h(q(t), \dot{q}(t), u(t)) & \geq 0, \quad t \in[0, \mathrm{~T}], \\
r\left(q(\mathrm{~T}), \dot{q}(\mathrm{~T}), q^{\mathrm{T}}, \dot{q}^{\mathrm{T}}\right) & =0 .
\end{aligned}
$$

The interval length $\mathrm{T}$ may either be fixed, or appear as degree of freedom in the optimization problem.

Definition 2.2. A curve $(q, u) \in C^{1,1}([0, T], Q) \times L^{\infty}([0, T], U)$ is feasible, if it fulfills the constraints (2.3b)(2.3e). The set of all feasible curves is the feasible set of Problem 2.1. A feasible curve $\left(q^{*}, u^{*}\right)$ is an optimal solution of Problem 2.1, if $J\left(q^{*}, u^{*}\right) \leq J(q, u)$ for all feasible curves $(q, u)$. An feasible curve $\left(q^{*}, u^{*}\right)$ is a local optimal solution, if $J\left(q^{*}, u^{*}\right) \leq J(q, u)$ in a neighborhood of $\left(q^{*}, u^{*}\right)$. The function $q^{*}$ is called (locally) optimal trajectory, and $u^{*}$ is the (locally) optimal control.

The Hamiltonian optimal control problem. We now formulate the problem using the Hamiltonian variant for the system dynamics. This is equivalent to the Lagrangian formulation as we have seen in Section 1.1 on the Legendre transform with forces. For a set $\mathcal{R}=\{(q, \dot{q}) \in T Q \mid g(q, \dot{q}) \geq 0\}$ determined via a constraint $g$ : $T Q \rightarrow \mathbb{R}^{n_{g}}$ on $T Q$ we obtain the corresponding set in the cotangent bundle as $\tilde{\mathcal{R}}=\left\{(q, p) \in T^{*} Q \mid \tilde{g}(q, p) \geq 0\right\}$ with $\tilde{g}=g \circ(\mathbb{F} L)^{-1}$. Analogously, we define $\tilde{J}, \tilde{C}, \tilde{\Phi}, \tilde{h}$, and $\tilde{r}$ such that the optimal control problem in the Hamiltonian formulation reads as follows:

Problem 2.3 (Hamiltonian optimal control problem (HOCP)).

$$
\begin{aligned}
\min _{q, p, u} \tilde{J}(q, p, u) & =\int_{0}^{\mathrm{T}} \tilde{C}(q(t), p(t), u(t)) \mathrm{d} t+\tilde{\Phi}(q(\mathrm{~T}), p(\mathrm{~T})) \\
\dot{q}(t) & =\nabla_{p} H(q(t), p(t)), \\
\dot{p}(t) & =-\nabla_{q} H(q(t), p(t))+f_{H}(q(t), p(t), u(t)), \\
\text { subject to }(q(0), p(0)) & =\left(q^{0}, p^{0}\right), \\
\tilde{h}(q(t), p(t), u(t)) & \geq 0, \quad t \in[0, \mathrm{~T}] \\
\tilde{r}\left(q(\mathrm{~T}), p(\mathrm{~T}), q^{\mathrm{T}}, p^{\mathrm{T}}\right) & =0,
\end{aligned}
$$

where $\left(q^{\mathrm{T}}, p^{\mathrm{T}}\right)=\mathbb{F} L\left(q^{\mathrm{T}}, \dot{q}^{\mathrm{T}}\right), p(0)=D_{2} L(q(0), \dot{q}(0)), p^{0}=D_{2} L\left(q^{0}, \dot{q}^{0}\right)$ and $\tilde{\Phi}=\Phi \circ(\mathbb{F} L)^{-1}$ etc., and the minimization is over $q \in C^{1,1}([0, \mathrm{~T}], Q)=W^{2, \infty}([0, \mathrm{~T}], Q), p \in W^{1, \infty}\left([0, \mathrm{~T}], T_{q}^{*} Q\right)$ and $u \in L^{\infty}([0, \mathrm{~T}], U)$. 
Necessary optimality conditions. In this paragraph, we derive necessary conditions for the optimality of a solution $\left(x^{*}, u^{*}\right)$ with $x=(q, p)$ to Problems 2.1 and 2.3. Since we need $\left(x^{*}, u^{*}\right) \in W^{2, \infty}\left([0, T], T^{*} Q\right) \times$ $W^{1, \infty}([0, \mathrm{~T}], U)$ later on we use the same smoothness assumption for the derivation. In addition, we restrict ourselves to the case of problems with the controls pointwise constrained to the (nonempty) set $U=\{u \in$ $\left.\mathbb{R}^{n_{u}} \mid h(u) \geq 0\right\}$ and fixed final time T. With $f(x, u)=\left(\nabla_{p} H(q, p),-\nabla_{q} H(q, p)+f_{H}(q, p, u)\right)$ we can rewrite (2.4) as

$$
\min _{\substack{x \in W^{2, \infty}\left([0, \mathrm{~T}], T^{*} Q\right) \\ u \in W^{1, \infty}([0, \mathrm{~T}], U)}} \tilde{J}(x, u)=\int_{0}^{\mathrm{T}} \tilde{C}(x(t), u(t)) \mathrm{d} t+\Phi(x(\mathrm{~T}))
$$

subject to $\dot{x}=f(x, u), x(0)=x_{0}, u(t) \in U$ for $t \in[0, \mathrm{~T}]$ and $\tilde{r}\left(x(\mathrm{~T}), x^{T}\right)=0$. Necessary conditions for optimality of solution trajectories $\eta(\cdot)=(x(\cdot), u(\cdot))$ can be derived based on variations of an augmented cost function, the Lagrangian of the system:

$$
\mathcal{L}(\eta, \lambda)=\int_{0}^{\mathrm{T}} \tilde{C}(x(t), u(t))+\lambda^{T}(t) \cdot(\dot{x}-f(x(t), u(t))) \mathrm{d} t+\Phi(x(\mathrm{~T})),
$$

where $\lambda \in W^{2, \infty}\left([0, T], \mathbb{R}^{n_{x}}\right)$ is the adjoint variable or the costate. A point $\left(\eta^{*}, \lambda^{*}\right)$ is a saddle point of (2.6), if $\mathcal{L}\left(\eta, \lambda^{*}\right) \leq \mathcal{L}\left(\eta^{*}, \lambda^{*}\right) \leq \mathcal{L}\left(\eta^{*}, \lambda\right)$ for all $\eta$ and $\lambda$. The function $\mathcal{H}(x, u, \lambda):=-\tilde{C}(x, u)+\lambda^{T} \cdot f(x, u)$ is called the Hamiltonian of the optimal control problem. When setting variations of $\mathcal{L}$ with respect to $\eta$ and $\lambda$ to zero, the resulting Euler-Lagrange equations provide necessary optimality condition for the optimal control problem (2.5). Formally, one obtains the following celebrated theorem (cf. [74]):

Theorem 2.4 (Pontryagin maximum principle). Let $\left(x^{*}, u^{*}\right) \in W^{2, \infty}\left([0, \mathrm{~T}], T^{*} Q\right) \times W^{1, \infty}([0, \mathrm{~T}], U)$ be an optimal solution to (2.5). Then there exists a function $\lambda \in W^{2, \infty}\left([0, \mathrm{~T}], \mathbb{R}^{n_{x}}\right)$ and a vector $\alpha \in \mathbb{R}^{n_{r}}$ such that

$$
\mathcal{H}\left(x^{*}(t), u^{*}(t), \lambda(t)\right)=\max _{u \in U} \mathcal{H}(x(t), u, \lambda(t)) \quad t \in[0, \mathrm{~T}],
$$

and $\lambda$ solves the following initial value problem:

$$
\lambda(\mathrm{T})=\nabla_{x} \Phi\left(x^{*}(\mathrm{~T})\right)-\nabla_{x} \tilde{r}\left(x^{*}(\mathrm{~T}), x^{\mathrm{T}}\right) \alpha, \quad \dot{\lambda}=-\nabla_{x} \mathcal{H}\left(x^{*}, u^{*}, \lambda\right) .
$$

\subsection{The discrete setting}

For the numerical solution we need a discretized version of Problem 2.1. To this end we formulate an optimal control problem for the discrete mechanical system described by discrete variational mechanics introduced in Section 1.2. In Section 2.3 we show how the optimal control problem for the continuous and the discrete mechanical system are related. To obtain a discrete formulation, we replace each expression in (2.3) by its discrete counterpart in terms of discrete variational mechanics. As described in Section 1.2, we replace the state space $T Q$ of the system by $Q \times Q$ and a path $q:[0, T] \rightarrow Q$ by a discrete path $q_{d}:\{0, h, 2 h, \ldots, N h=T\} \rightarrow Q$ with $q_{k}=q_{d}(k h)$. Analogously, the continuous control path $u:[0, \mathrm{~T}] \rightarrow U$ is replaced by a discrete control path $u_{d}: \Delta \tilde{t} \rightarrow U$ (writing $\left.u_{k}=\left(u_{d}\left(k h+c_{\ell} h\right)\right)_{\ell=1}^{s} \in U^{s}\right)$.

The discrete Lagrange-d'Alembert principle. Based on this discretization, the action integral in (2.2) is approximated on a time slice $[k h,(k+1) h]$ by the discrete Lagrangian $L_{d}: Q \times Q \rightarrow \mathbb{R}, L_{d}\left(q_{k}, q_{k+1}\right) \approx$ $\int_{k h}^{(k+1) h} L(q(t), \dot{q}(t)) \mathrm{d} t$, and likewise the virtual work by the left and right discrete forces, $f_{k}^{-} \cdot \delta q_{k}+f_{k}^{+} \cdot \delta q_{k+1} \approx$ $\int_{k h}^{(k+1) h} f_{L}(q(t), \dot{q}(t), u(t)) \cdot \delta q(t) \mathrm{d} t$, where $f_{k}^{-}, f_{k}^{+} \in T^{*} Q$. As introduced in equation (1.8), the discrete version of the Lagrange-d'Alembert principle (2.2) requires one to find discrete paths $\left\{q_{k}\right\}_{k=0}^{N}$ such that for all variations $\left\{\delta q_{k}\right\}_{k=0}^{N}$ with $\delta q_{0}=\delta q_{N}=0$, one has the discrete Lagrange-d'Alembert principle (1.8), or, equivalently, the forced discrete Euler-Lagrange equations (1.9). 
Boundary conditions. In the next step, we need to incorporate the boundary conditions $q(0)=q^{0}, \dot{q}(0)=\dot{q}^{0}$ and $r\left(q(\mathrm{~T}), \dot{q}(\mathrm{~T}), q^{\mathrm{T}}, \dot{q}^{\mathrm{T}}\right)=0$ into the discrete description. Those on the configuration level can be used as constraints in a straightforward way as $q_{0}=q^{0}$. However, since in the present formulation velocities are approximated in a time interval $\left[t_{k}, t_{k+1}\right]$ (as opposed to an approximation at the time nodes), the velocity conditions have to be transformed to conditions on the conjugate momenta. These are defined at each time node using the discrete Legendre transform. The presence of forces at the time nodes has to be incorporated into that transformation leading to the forced discrete Legendre transforms $\mathbb{F}^{f^{-}} L_{d}$ and $\mathbb{F}^{f+} L_{d}$ defined in (1.10). Using the standard Legendre transform $\mathbb{F} L: T Q \rightarrow T^{*} Q,(q, \dot{q}) \mapsto(q, p)=\left(q, D_{2} L(q, \dot{q})\right)$ leads to the discrete initial constraint on the conjugate momentum

$$
D_{2} L\left(q^{0}, \dot{q}^{0}\right)+D_{1} L_{d}\left(q_{0}, q_{1}\right)+f_{d}^{-}\left(q_{0}, q_{1}, u_{0}\right)=0 .
$$

As shown in the previous section, we can transform the boundary condition from a formulation with configuration and velocity to a formulation with configuration and conjugate momentum. Thus, instead of considering a discrete version of the final time constraint $r$ on $T Q$ we use a discrete version of the final time constraint $\tilde{r}$ on $T^{*} Q$. We define the discrete boundary condition on the configuration level to be $r_{d}: Q \times Q \times U^{s} \times T Q \rightarrow \mathbb{R}^{n_{r}}$,

$$
r_{d}\left(q_{N-1}, q_{N}, u_{N-1}, q^{\mathrm{T}}, \dot{q}^{\mathrm{T}}\right)=\tilde{r}\left(\mathbb{F}^{f+} L_{d}\left(q_{N-1}, q_{N}, u_{N-1}\right), \mathbb{F} L\left(q^{\mathrm{T}}, \dot{q}^{\mathrm{T}}\right)\right),
$$

i.e. we used $\left(q_{N}, p_{N}\right)=\mathbb{F}^{f+} L_{d}\left(q_{N-1}, q_{N}, u_{N-1}\right)$ and $\left(q^{\mathrm{T}}, p^{\mathrm{T}}\right)=\mathbb{F} L\left(q^{\mathrm{T}}, \dot{q}^{\mathrm{T}}\right)$, that is $p_{N}=D_{2} L_{d}\left(q_{N-1}, q_{N}\right)+$ $f_{d}^{+}\left(q_{N-1}, q_{N}, u_{N-1}\right)$ and $p^{\mathrm{T}}=D_{2} L\left(q^{\mathrm{T}}, \dot{q}^{\mathrm{T}}\right)$. Notice that for the simple final velocity constraint $\dot{q}(\mathrm{~T})-\dot{q}^{\mathrm{T}}=0$, we obtain for the transformed condition on the momentum level $\tilde{r}\left(q(\mathrm{~T}), p(\mathrm{~T}), q^{\mathrm{T}}, p^{\mathrm{T}}\right)=p(\mathrm{~T})-p^{\mathrm{T}}$ the discrete constraint

$$
-D_{2} L\left(q^{\mathrm{T}}, \dot{q}^{\mathrm{T}}\right)+D_{2} L_{d}\left(q_{N-1}, q_{N}\right)+f_{d}^{+}\left(q_{N-1}, q_{N}, u_{N-1}\right)=0
$$

which together with equation (2.8) constitute the boundary constraints on momentum level.

Discrete path constraints. Opposed to the final time constraint we approximate the path constraint in (2.3d) on each time interval $\left[t_{k}, t_{k+1}\right]$ rather than at each time node. Thus, we maintain the formulation on the velocity level and replace the continuous path constraint $h(q(t), \dot{q}(t), u(t)) \geq 0$ by a discrete path constraint $h_{d}: Q \times Q \times U^{s} \rightarrow \mathbb{R}^{s n_{h}}$ which suitably approximate the continuous constraint pointwise (see Sect. 2.4) with $h_{d}\left(q_{k}, q_{k+1}, u_{k}\right) \geq 0, k=0, \ldots, N-1$.

Discrete objective function. Similar to the Lagrangian we approximate the objective functional in (2.1) on the time slice $[k h,(k+1) h]$ by $C_{d}\left(q_{k}, q_{k+1}, u_{k}\right) \approx \int_{k h}^{(k+1) h} C(q(t), \dot{q}(t), u(t)) \mathrm{d} t$. Analogously to the final time constraint, we approximate the final condition via a discrete version $\Phi_{d}: Q \times Q \times U^{s} \rightarrow \mathbb{R}$ yielding the discrete objective function

$$
J_{d}\left(q_{d}, u_{d}\right)=\sum_{k=0}^{N-1} C_{d}\left(q_{k}, q_{k+1}, u_{k}\right)+\Phi_{d}\left(q_{N-1}, q_{N}, u_{N-1}\right) .
$$

The discrete optimal control problem. In summary, after performing the above discretization steps, one is faced with the following discrete optimal control problem.

Problem 2.5 (discrete Lagrangian optimal control problem).

$$
\min _{\left(q_{d}, u_{d}\right) \in \mathcal{P}_{d}(Q) \times \mathcal{P}_{d}(U)} J_{d}\left(q_{d}, u_{d}\right)
$$

subject to

$$
q_{0}=q^{0},
$$

$$
\begin{aligned}
D_{2} L\left(q^{0}, \dot{q}^{0}\right)+D_{1} L_{d}\left(q_{0}, q_{1}\right)+f_{0}^{-}=0, & \\
D_{2} L_{d}\left(q_{k-1}, q_{k}\right)+D_{1} L_{d}\left(q_{k}, q_{k+1}\right)+f_{k-1}^{+}+f_{k}^{-}=0, & k=1, \ldots, N-1, \\
h_{d}\left(q_{k}, q_{k+1}, u_{k}\right) \geq 0, & k=0, \ldots, N-1, \\
r_{d}\left(q_{N-1}, q_{N}, u_{N-1}, q^{\mathrm{T}}, \dot{q}^{\mathrm{T}}\right) & =0 .
\end{aligned}
$$


Recall that the $f_{k}^{ \pm}$are dependent on $u_{k} \in U^{s}$. To incorporate a free final time $\mathrm{T}$ as in the continuous setting, the step size $h$ appears as a degree of freedom within the optimization problem. However, in the following formulations and considerations we restrict ourselves to the case of fixed final time T and thus fixed step size $h$. Special case: fixed boundary conditions. Consider the special case of a problem with fixed initial and final configuration and velocities and without path constraints, i.e. consider Problem 2.1 without the constraint (2.3d) and $r(q(\mathrm{~T}), \dot{q}(\mathrm{~T}))=\left(q(\mathrm{~T})-q^{\mathrm{T}}, \dot{q}(\mathrm{~T})-\dot{q}^{\mathrm{T}}\right)$. A straightforward way to derive initial and final constraints for the conjugate momenta rather than for the velocities from the variational principle directly is stated in the following proposition:

Proposition 2.6. With $\left(q^{0}, p^{0}\right)=\mathbb{F} L\left(q^{0}, \dot{q}^{0}\right)$ and $\left(q^{T}, p^{T}\right)=\mathbb{F} L\left(q^{N}, \dot{q}^{N}\right)$ equations (2.3b), (2.3c) and (2.3e) are equivalent to the following principle with free initial and final variation and with augmented Lagrangian

$$
\delta\left(\int_{0}^{T} L(q(t), \dot{q}(t)) \mathrm{d} t+p^{0}\left(q(0)-q^{0}\right)-p^{T}\left(q(T)-q^{T}\right)\right)+\int_{0}^{T} f_{L}(q(t), \dot{q}(t), u(t)) \cdot \delta q(t) \mathrm{d} t=0 .
$$

Proof. Variations of $(2.3 \mathrm{~b})$ with respect to $q$ and zero initial and final variation $\delta q(0)=\delta q(\mathrm{~T})=0$ together with $(2.3 \mathrm{c}),(2.3 \mathrm{e})$ yield

$$
\begin{aligned}
& \frac{\mathrm{d}}{\mathrm{d} t} \frac{\partial}{\partial \dot{q}} L(q(t), \dot{q}(t))-\frac{\partial}{\partial q} L(q(t), \dot{q}(t))=f_{L}(q(t), \dot{q}(t), u(t)), \\
& q(0)=q^{0}, \dot{q}(0)=\dot{q}^{0}, q(\mathrm{~T})=q^{\mathrm{T}}, \dot{q}(\mathrm{~T})=\dot{q}^{\mathrm{T}} .
\end{aligned}
$$

On the other hand variations of (2.11) with respect to $q$ and $\lambda=\left(p^{0}, p^{T}\right)$ with free initial and final variation lead to

$$
\begin{aligned}
& \frac{\mathrm{d}}{\mathrm{d} t} \frac{\partial}{\partial \dot{q}} L(q(t), \dot{q}(t))-\frac{\partial}{\partial q} L(q(t), \dot{q}(t))=f_{L}(q(t), \dot{q}(t), u(t)), \\
& q(0)=q^{0}, q(\mathrm{~T})=q^{\mathrm{T}},\left.\frac{\partial}{\partial \dot{q}} L(q(t), \dot{q}(t))\right|_{t=0}=p^{\mathrm{T}},\left.\quad \frac{\partial}{\partial \dot{q}} L(q(t), \dot{q}(t))\right|_{t=\mathrm{T}}=p^{0} .
\end{aligned}
$$

The Legendre transform applied to the velocity boundary equations in (2.12b) gives the corresponding momenta boundary equations $(2.13 \mathrm{~b})$.

On the discrete level we derive the optimal control problem for fixed initial and final configurations and velocities in an equivalent way. Thus, we consider the discrete principle with discrete augmented Lagrangian

$$
\delta\left(\sum_{k=0}^{N-1} L_{d}\left(q_{k}, q_{k+1}\right)+p^{0}\left(q_{0}-q^{0}\right)-p^{\mathrm{T}}\left(q_{N}-q^{\mathrm{T}}\right)\right)+\sum_{k=0}^{N-1}\left[f_{k}^{-} \cdot \delta q_{k}+f_{k}^{+} \cdot \delta q_{k+1}\right]=0,
$$

which, with free initial and final variation $\delta q_{0}$ and $\delta q_{N}$, respectively, is equivalent to

$$
\begin{array}{r}
\delta \sum_{k=0}^{N-1} L_{d}\left(q_{k}, q_{k+1}\right)+\sum_{k=0}^{N-1}\left[f_{k}^{-} \cdot \delta q_{k}+f_{k}^{+} \cdot \delta q_{k+1}\right]=0, \\
q_{0}=q^{0}, q_{N}=q^{\mathrm{T}}, p^{0}+D_{1} L_{d}\left(q_{0}, q_{1}\right)+f_{0}^{-}=0,-p^{\mathrm{T}}+D_{2} L_{d}\left(q_{N-1}, q_{N}\right)+f_{N-1}^{+}=0,
\end{array}
$$

where the third and fourth equations in (2.15b) are exactly the discrete initial and final velocity constraints derived in the remark containing equation (2.9) with $p^{0}=D_{2} L\left(q^{0}, \dot{q}^{0}\right)$ and $p^{\mathrm{T}}=D_{2} L\left(q^{\mathrm{T}}, \dot{q}^{\mathrm{T}}\right)$.

We note that this derivation of the discrete initial and final conditions directly gives the same formulation that we found before by first transforming the boundary condition on the momentum level $T^{*} Q$ and then formulating the corresponding discrete constraints on $Q \times Q \times U^{s}$. 
Necessary optimality conditions. The system (2.10) results in a constrained nonlinear optimization problem, also called a nonlinear programming problem, that is an objective function has to be minimized subject to algebraic equality and inequality constraints. Let $\xi$ be the set of parameters introduced by the discretization of an infinite dimensional optimal control problem. Then, the nonlinear programming problem (NLP) to be solved is

$$
\min _{\xi} \varphi(\xi), \quad \text { subject to } a(\xi)=0, b(\xi) \geq 0,
$$

where $\varphi: \mathbb{R}^{n} \rightarrow \mathbb{R}, a: \mathbb{R}^{n} \rightarrow \mathbb{R}^{m}$, and $b: \mathbb{R}^{n} \rightarrow \mathbb{R}^{p}$ are continuously differentiable. We briefly summarize some terminology: A feasible point is a point $\xi \in \mathbb{R}^{n}$ that satisfies $a(\xi)=0$ and $b(\xi) \geq 0$. A (strict) local minimum of (2.16) is a feasible point $\xi^{*}$ with $\varphi\left(\xi^{*}\right) \leq \varphi(\xi)\left(\varphi\left(\xi^{*}\right)<\varphi(\xi)\right)$ for all feasible points $\xi$ in a neighborhood of $\xi^{*}$. Active inequality constraints $b^{\text {act }}(\xi)$ at a feasible point $\xi$ are those components $b_{j}(\xi)$ of $b(\xi)$ with $b_{j}(\xi)=0$. Subsuming the equality constraints and the active inequalities at a point $\xi$ (known as active set) in a combined vector function gives the active constraints as $\tilde{a}(\xi):=\left(a(\xi), b^{\text {act }}(\xi)\right)$. The active set may be different at different feasible points. Feasible points $\xi$ that satisfy the condition that the Jacobian of the active constraints, $\nabla \tilde{a}(\xi)^{T}$, has full rank are denoted as regular points.

To investigate local optimality in the presence of constraints, we introduce the Lagrangian multiplier vectors $\lambda \in \mathbb{R}^{m}$ and $\mu \in \mathbb{R}^{p}$, that are also called adjoint variables, and we define the Lagrangian function $\tilde{\mathcal{L}}$ by

$$
\tilde{\mathcal{L}}(\xi, \lambda, \mu):=\varphi(\xi)-\lambda^{T} a(\xi)-\mu^{T} b(\xi)
$$

The following variant of the Karush-Kuhn-Tucker necessary conditions for local optimality of a point $\xi^{*}$ have been derived first by Karush in 1939 [41] and independently by Kuhn and Tucker in 1951 [49]. For brevity, we restrict our attention to regular points only.

Theorem 2.7 (Karush-Kuhn-Tucker conditions (KKT)). If a regular point $\xi^{*} \in \mathbb{R}^{n}$ is a local optimum of the NLP problem (2.16), then there exist unique Lagrange multiplier vectors $\lambda^{*} \in \mathbb{R}^{m}$ and $\mu^{*} \in \mathbb{R}^{p}$ such that the triple $\left(\xi^{*}, \lambda^{*}, \mu^{*}\right)$ satisfies the following necessary conditions:

$$
\nabla_{\xi} \tilde{\mathcal{L}}\left(\xi^{*}, \lambda^{*}, \mu^{*}\right)=0, \quad a\left(\xi^{*}\right)=0, \quad b\left(\xi^{*}\right) \geq 0, \quad \mu^{*} \geq 0, \quad \mu_{j}^{*} b_{j}\left(\xi^{*}\right)=0, \quad j=1, \ldots, p .
$$

A triple $\left(\xi^{*}, \lambda^{*}, \mu^{*}\right)$ that satisfies these conditions is called a Karush-Kuhn-Tucker point (KKT point).

\subsection{The discrete $v s$. the continuous problem}

This section gives an interpretation of the discrete problem as an approximation to the continuous one. In addition, we identify certain structural properties that the discrete problem inherits from the continuous one. We determine the consistency order of the discrete scheme and establish a result on the convergence of the discrete solution as the step size goes to zero.

The place of DMOC amongst solution methods for optimal control problems. In Figure 2 we present schematically different discretization strategies for optimal control problems:

- In an indirect method, starting with an objective function and the Lagrange-d'Alembert principle we obtain via two variations (the first for the derivation of the Euler-Lagrange equations and the second for the derivation of the necessary optimality conditions) the Pontryagin maximum principle. The resulting boundary value problem is then solved numerically, e.g. by gradient methods $[11,16,17,43,70$, 81], multiple shooting $[7,12,18,24,32,42]$ or collocation $[1,2,19]$.

- In a direct approach, starting form the Euler-Lagrange equations we directly transform the problem into a restricted finite dimensional optimization problem by discretizing the differential equation. Common methods like e.g. shooting [48], multiple shooting [8], or collocation methods [82], rely on a direct integration of the associated ordinary differential equations or on its fulfillment at certain grid points (see also $[3,76]$ for an overview of the current state of the art). The resulting finite dimensional nonlinear constrained optimization problem can be solved by standard nonlinear optimization techniques 


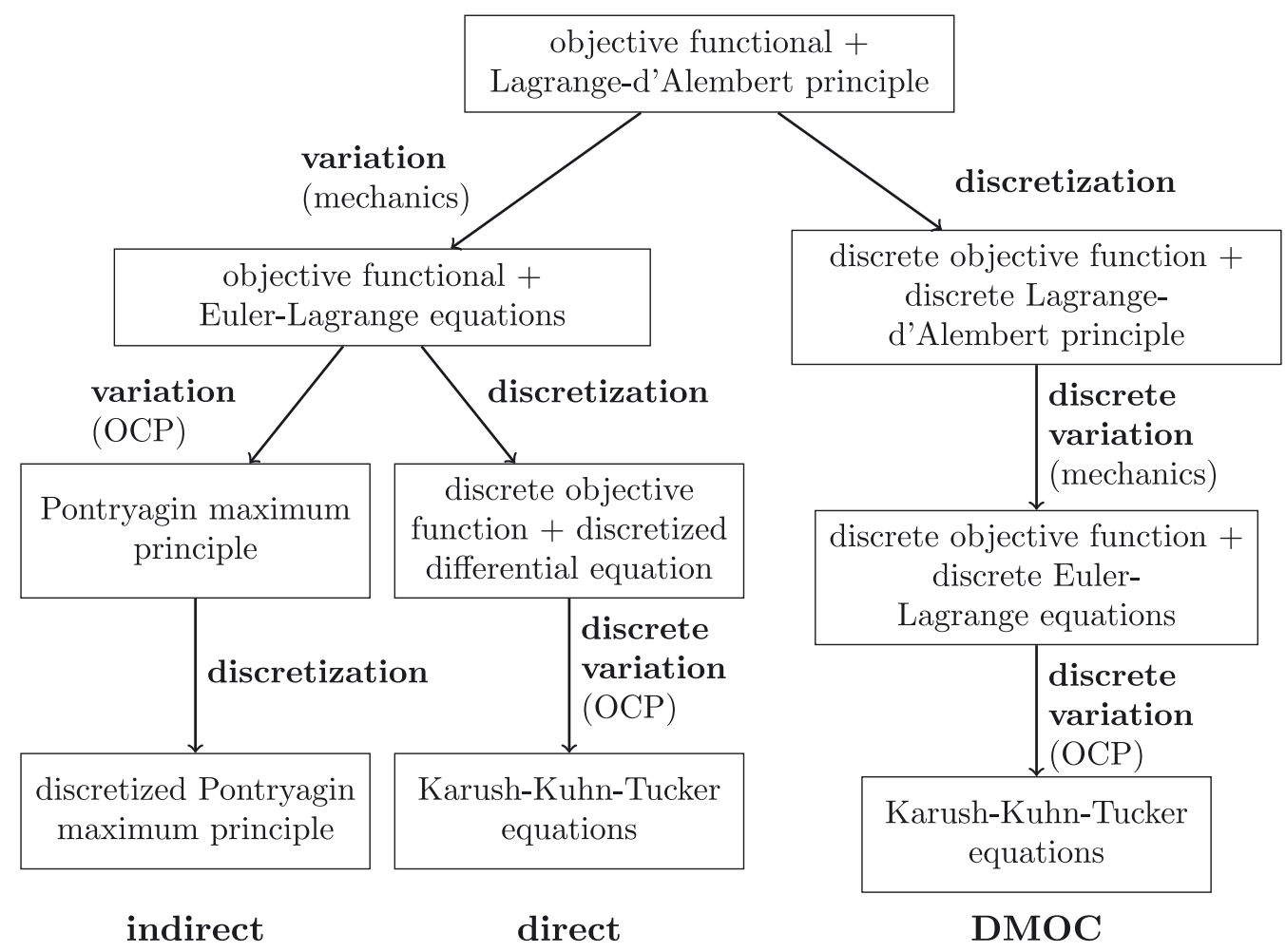

FIGURE 2. Optimal control for mechanical systems: the order of variation and discretization for deriving the necessary optimality conditions.

like sequential quadratic programming [31,75]. Implementations are found in software packages like DIRCOL [83], SOCS [4], or MUSCOD [54].

- In the $D M O C$ approach, rather than discretizing the differential equations arising from the Lagranged'Alembert principle, we discretize in the earliest stage, namely already on the level of the variational principle. Then, we consider variations only on the discrete level to derive the restricted optimization problem and its necessary optimality conditions.

This approach derived via the concept of discrete mechanics leads to a special discretization of the system equations based on variational integrators, which are dealt with in detail in [64]. Thus, the discrete optimal control problem inherits special properties exhibited by variational integrators. In the following, we specify particular important properties and phenomena of variational integrators and try to translate their meaning into the optimal control context.

Preservation of momentum maps. If the discrete system, obtained by applying variational integration to a mechanical system, inherits the same symmetry groups as the continuous system, the corresponding discrete momentum maps are preserved. For the forced case the same statement holds, if the forcing is orthogonal to the group action (see Thm. 1.3). On the one hand, this means for the optimal control problem, that if the control force is orthogonal to the group action, our discretization leads to a discrete system, for which the corresponding momentum map is preserved. On the other hand, in the case of the forcing not being orthogonal to the group action, the forced discrete Noether's theorem provides an exact coherence between the change 
in angular momentum and the applied control force via (see Sect. 3.2 for examples)

$$
\left[\mathbf{J}_{L_{d}}^{f+} \circ\left(F_{L_{d}}^{u_{d}}\right)^{N-1}-\mathbf{J}_{L_{d}}^{f-}\right]\left(q_{0}, q_{1}\right) \cdot \xi=\sum_{k=0}^{N-1} f_{d}^{u_{k}}\left(q_{k}, q_{k+1}\right) \cdot \xi_{Q \times Q}\left(q_{k}, q_{k+1}\right) .
$$

Conservation of a modified energy. Variational integrators are symplectic, which implies that a certain modified energy is conserved (see for example [30]). This is an important property if the long time behavior of dynamical systems is considered. For the case of the optimal control of systems with long maneuver time such as low thrust space missions, it would therefore be interesting to investigate the relation between a modified energy and the virtual work. However, this has not been considered within this paper.

Implementation. Rather than using a configuration-momentum implementation of variational integrators as proposed in [64], we stay on $Q \times Q$. That means we just determine the optimal trajectory for the configuration and the control forces and reconstruct the corresponding momenta and velocities via the forced discrete Legendre transforms. This yields computational savings. A more detailed description of the computational savings compared to standard discretizations for optimal control problems is given in Remark 2.11.

\subsection{The correspondence with Runge-Kutta discretizations}

In this section we are going to show that the discretization derived via the discrete Lagrange-d'Alembert principle is equivalent to one resulting from a finite difference discretization of the associated Hamiltonian system via a symplectic partitioned Runge-Kutta scheme.

Symplectic partitioned Runge-Kutta methods. As shown in [64] (Thm. 2.6.1), the discrete Hamiltonian map generated by the discrete Lagrangian is a symplectic partitioned Runge-Kutta method. As we will show, a similar statement is true for discrete Hamiltonian maps with forces. The resulting method is still a partitioned Runge-Kutta method, but no longer symplectic in the original sense since the symplectic form is not preserved anymore due to the presence of control forces. However, we still denote it as a symplectic method having in mind that the symplectic form is preserved only in absence of external control forces.

A partitioned Runge-Kutta method for the regular forced Lagrangian system $\left(L, f_{L}\right)$ is a map $T^{*} Q \times U^{s} \rightarrow$ $T^{*} Q$ specified by coefficients $b_{i}, a_{i j}, \tilde{b}_{i}, \tilde{a}_{i j}, i=1, \ldots, s$, and defined by $\left(q_{0}, p_{0}, u_{0}\right) \mapsto\left(q_{1}, p_{1}\right)$, where

$$
\begin{aligned}
q_{1} & =q_{0}+h \sum_{j=1}^{s} b_{j} \dot{Q}_{j}, & p_{1} & =p_{0}+h \sum_{j=1}^{s} \tilde{b}_{j} \dot{P}_{j}, \\
Q_{i} & =q_{0}+h \sum_{j=1}^{s} a_{i j} \dot{Q}_{j}, & P_{i} & =p_{0}+h \sum_{j=1}^{s} \tilde{a}_{i j} \dot{P}_{j}, \\
P_{i} & =\frac{\partial L}{\partial \dot{q}}\left(Q_{i}, \dot{Q}_{i}\right), & \dot{P}_{i} & =\frac{\partial L}{\partial q}\left(Q_{i}, \dot{Q}_{i}\right)+f_{L}\left(Q_{i}, \dot{Q}_{i}, U_{i}\right),
\end{aligned}
$$

$i=1, \ldots, s$, where the points $\left(Q_{i}, P_{i}\right)$ are known as the internal stages and $U_{i}$ are the control samples given by $U_{i}=u_{0 i}=u_{d}\left(t_{0}+c_{i} h\right)$. For $a_{i j}=\tilde{a}_{i j}$ and $b_{i}=\tilde{b}_{i}$ the partitioned Runge-Kutta method is a Runge-Kutta method. The method is symplectic (that is, it preserves the canonical symplectic form $\Omega$ on $T^{*} Q$ in absence of external forces) if the coefficients satisfy

$$
b_{i} \tilde{a}_{i j}+\tilde{b}_{j} a_{j i}=b_{i} \tilde{b}_{j} \quad \text { and } \quad b_{i}=\tilde{b}_{i}, \quad i, j=1, \ldots, s .
$$

Since discrete Lagrangian maps are symplectic, we can assume that we have coefficients satisfying (2.19) and write a discrete Lagrangian and discrete Lagrangian control forces that generate the corresponding symplectic partitioned Runge-Kutta method. Given points $\left(q_{0}, q_{1}\right) \in Q \times Q$, we can regard (2.18) as implicitly defining $p_{0}, p_{1}, Q_{i}, P_{i}, \dot{Q}_{i}$ and $\dot{P}_{i}$ for $i=1, \ldots, s$. Taking these to be defined as functions of $\left(q_{0}, q_{1}\right)$, we construct 
a discrete Lagrangian

$$
L_{d}\left(q_{0}, q_{1}, h\right)=h \sum_{i=1}^{s} b_{i} L\left(Q_{i}, \dot{Q}_{i}\right)
$$

and left and right discrete forces as

$$
f_{d}^{+}\left(q_{0}, q_{1}, u_{0}, h\right)=h \sum_{i=1}^{s} b_{i} f_{L}\left(Q_{i}, \dot{Q}_{i}, U_{i}\right) \cdot \frac{\partial Q_{i}}{\partial q_{1}}, \quad f_{d}^{-}\left(q_{0}, q_{1}, u_{0}, h\right)=h \sum_{i=1}^{s} b_{i} f_{L}\left(Q_{i}, \dot{Q}_{i}, U_{i}\right) \cdot \frac{\partial Q_{i}}{\partial q_{0}}
$$

For fixed $u_{0}$ the corresponding forced discrete Hamiltonian map is exactly the map $\left(q_{0}, p_{0}\right) \mapsto\left(q_{1}, p_{1}\right)$ which is the symplectic partitioned Runge-Kutta method for the forced Hamiltonian system (1.4). Straight forward to the proof for unforced systems in [64] (Thm. 2.6.1) we get:

Theorem 2.8. The discrete Hamiltonian map generated by the discrete Lagrangian (2.20) together with the discrete forces (2.21) is a partitioned Runge-Kutta method (which is symplectic in the unforced case).

Optimal control and Runge-Kutta discretizations. In this paragraph, we carry forward the results of the previous section into the context of optimal control problems. To formulate the optimal control problem for the discrete system in terms of Runge-Kutta discretizations, we have to give appropriate expressions for the boundary conditions, the path constraints, and the objective functional. Concerning the dynamics of the mechanical system, we have already seen that the discretization obtained via the discrete Lagrange-d'Alembert principle can be rewritten as a Runge-Kutta scheme for the corresponding mechanical system in terms of $(p, q)$ as in (2.18). Since we consider regular Lagrangians and regular Hamiltonians, we reformulate the last equation of (2.18) with the help of the Hamiltonian as

$$
\dot{Q}_{k i}=\frac{\partial H}{\partial p}\left(Q_{k i}, P_{k i}\right), \quad \dot{P}_{k i}=-\frac{\partial H}{\partial q}\left(Q_{k i}, P_{k i}\right)+f_{H}\left(Q_{k i}, P_{k i}, U_{k i}\right)
$$

where the additional index $i \in\{1, \ldots, s\}$ denotes the dependence of the intermediate state and control variables on the time interval $\left[t_{k}, t_{k+1}\right]$.

Boundary conditions. Due to a formulation of the optimal control problem within the Hamiltonian framework, we use the same formulation for the boundary constraint as the one for the continuous Hamiltonian optimal control Problem 2.3 evaluated at $\left(q_{N}, p_{N}\right)$, i.e. $\tilde{r}\left(q_{N}, p_{N}, q^{\mathrm{T}}, p^{\mathrm{T}}\right)=0$.

Discrete path constraints. Again we use the formulation in Problem 2.3 and enforce the path constraints to hold in each time step $\left(Q_{i}, P_{i}, U_{i}\right)$ as $\tilde{h}\left(Q_{k i}, P_{k i}, U_{k i}\right) \geq 0$ for $k=0, \ldots, N-1$ and $i=1, \ldots, s$.

Discrete objective function. We construct the discrete objective function in the same way as the discrete Lagrangian (2.20). However, corresponding to the Hamiltonian formulation evaluated in each substep $\left(Q_{i}, P_{i}, U_{i}\right)$, it now reads

$$
\tilde{J}_{d}\left(q_{d}, p_{d}, u_{d}\right)=\sum_{k=0}^{N-1} h \sum_{i=1}^{s} b_{i} \tilde{C}\left(Q_{k i}, P_{k i}, U_{k i}\right)+\tilde{\Phi}\left(q_{N}, p_{N}\right)
$$

where the final constraint holds for the node corresponding to the final time T. Combining terms, the discrete 
optimal control problem from the Hamiltonian point of view reads

$$
\begin{aligned}
& \min _{q_{d}, p_{d}, u_{d}} \tilde{J}_{d}\left(q_{d}, p_{d}, u_{d}\right) \\
& \text { subject to } \quad\left(q_{0}, p_{0}\right)=\left(q^{0}, p^{0}\right), \\
& q_{k+1}=q_{k}+h \sum_{j=1}^{s} b_{j} \dot{Q}_{k j}, \quad Q_{k i}=q_{k}+h \sum_{j=1}^{s} a_{i j} \dot{Q}_{k j}, \\
& p_{k+1}=p_{k}+h \sum_{j=1}^{s} \tilde{b}_{j} \dot{P}_{k j}, \quad P_{k i}=p_{k}+h \sum_{j=1}^{s} \tilde{a}_{i j} \dot{P}_{k j}, \\
& \dot{Q}_{k i}=\frac{\partial H}{\partial p}\left(Q_{k i}, P_{k i}\right), \quad \dot{P}_{k i}=-\frac{\partial H}{\partial q}\left(Q_{k i}, P_{k i}\right)+f_{H}\left(Q_{k i}, P_{k i}, U_{k i}\right),
\end{aligned}
$$

$\tilde{h}\left(Q_{k i}, P_{k i}, U_{k i}\right) \geq 0$ for $k=0, \ldots, N-1, i=1, \ldots, s$ and $\tilde{r}\left(q_{N}, p_{N}, q^{\mathrm{T}}, p^{\mathrm{T}}\right)=0$. Problem (2.22) is the finite dimensional optimization problem resulting from the Hamiltonian optimal control Problem 2.3 that is discretized via a symplectic partitioned Runge-Kutta scheme.

Theorem 2.9 (equivalence). Given a discrete Lagrangian and discrete forces as defined in (2.20) and (2.21), respectively. Then, the discrete Lagrangian optimal control problem defined in (2.10) and the problem (2.22) resulting from discretizing the Hamiltonian optimal control problem by a symplectic partitioned Runge-Kutta scheme are equivalent in the sense that both problems have the same set of solutions.

Proof. Assume $\left(q_{d}^{*}, u_{d}^{*}\right)$ is a solution of (2.10). By using the discrete Legendre transform we obtain an optimal solution in terms of the discrete momenta as $\left(q_{d}^{*}, p_{d}^{*}, u_{d}^{*}\right)$. To prove that $\left(q_{d}^{*}, p_{d}^{*}, u_{d}^{*}\right)$ is also an optimal solution for problem $(2.22)$ we have to show feasibility and optimality. Theorem 2.8 and application of the forced discrete Legendre transform to the boundary conditions give us the equivalence of equations (2.10b)-(2.10d), (2.10f) and $(2.22 \mathrm{c})-(2.22 \mathrm{e})$ and $\tilde{r}\left(q_{N}, p_{N}, q^{\mathrm{T}}, p^{\mathrm{T}}\right)=0$. For the choice of the discretizations of the curves $q(t)$ and $u(t)$ the inequality condition $(2.10 \mathrm{e})$ reads as $h\left(Q_{k i}, Q_{k, i+1}, U_{k i}\right) \geq 0$ for $i=1, \ldots, s-1$ and $h\left(Q_{k s}, Q_{k+1,0}, U_{k s}\right) \geq 0$. Again, due to the forced discrete Legendre transform $h$ and $\tilde{h}$ determine the same solution set. This makes $\left(q_{d}^{*}, p_{d}^{*}, u_{d}^{*}\right)$ a feasible solution of the problem (2.22). Optimality holds due to $\tilde{J}_{d}\left(q_{d}^{*}, p_{d}^{*}, u_{d}^{*}\right)=J_{d}\left(q_{d}^{*}, u_{d}^{*}\right) \leq$ $J_{d}\left(q_{d}, u_{d}\right)=\tilde{J}_{d}\left(q_{d}, p_{d}, u_{d}\right)$ for all feasible points $\left(q_{d}, p_{d}\right) \in T^{*} \mathcal{P}_{d}(Q)$ and $u_{d} \in \mathcal{P}_{d}(U)$ (global optimality), or for all feasible points $\left(q_{d}, p_{d}, u_{d}\right)$ in a neighborhood of $\left(q_{d}^{*}, p_{d}^{*}, u_{d}^{*}\right)$ (local optimality). Analogous, we can show that an optimal solution $\left(q_{d}^{*}, p_{d}^{*}, u_{d}^{*}\right)$ of problem (2.22) is also an optimal solution for problem (2.10).

\section{Example 2.10.}

(a) With $b=1$ and $a=\frac{1}{2}$ we obtain the implicit midpoint rule as a symplectic Runge-Kutta scheme, that is the partitioned scheme reduces to a standard one-stage Runge-Kutta scheme. This is equivalent to the discretization derived via the Lagrangian approach with midpoint quadrature.

(b) The standard Lobatto IIIA-IIIB partitioned Runge-Kutta method is obtained by using the Lobatto quadrature for the discrete Lagrangian.

\section{Remark 2.11.}

1. The formulations based on the discrete Lagrange-d'Alembert principle on the one hand side and based on a Runge-Kutta discretization of the Hamiltonian system on the other hand side are equivalent in the sense that the same solution set is described. However, the Lagrangian approach uses less variables and less constraints: For $q \in \mathbb{R}^{n}$ and $N$ intervals of discretization we need $(N s+1) n$ configurations $q_{k}^{\nu}$, $k=0, \ldots, N-1, \nu=0, \ldots, s$ with $q_{k}^{s}=q_{k+1}^{0}, k=1, \ldots, N-1$ and $n N(s-1)+n(N-1)$ extended discrete Euler-Lagrange equations, so altogether, $(N s-1) n$ constraints excluding boundary conditions. The Runge-Kutta approach for the Hamiltonian system uses $2(N s+1) n$ configurations and momenta and $2 n N+2 n N(s-1)=2 n N s$ equality constraints. Thus, via the Runge-Kutta approach we obtain 
twice as many state variables and $(N s+1) n$ more equality constraints. Comparisons concerning the computational effort are presented in Section 3.2.2.

2. Another advantage of the variational approach over the symplectic Runge-Kutta discretization is that one does not have to handle any conditions on the coefficients (such as condition (2.19)) to enforce symplecticity. The symplecticity of the DMOC discretization results naturally from the variational structure of this approach.

\subsection{Transformation to Mayer form}

Continuous system. Within the previous sections we introduced optimal control problems in Bolza form, in which the objective functional consists of a cost functional of integral form and a final point constraint. For the error analysis in Sections 2.6 and 2.7 it will be useful to transform the problem into Mayer form, in which the objective functional consists of the final point constraint only. To this end we introduce a new state variable as

$$
z(t):=\int_{0}^{t} C(q(\tau), \dot{q}(\tau), u(\tau)) \mathrm{d} \tau, \quad 0 \leq t \leq \mathrm{T}
$$

resp. $y(t):=\int_{0}^{t} \tilde{C}(q(\tau), p(\tau), u(\tau)) \mathrm{d} \tau, 0 \leq t \leq \mathrm{T}$ for the Hamiltonian form. By extension of the state space from $T Q$ to $T Q \times \mathbb{R}$, and from $T^{*} Q$ to $T^{*} Q \times \mathbb{R}$, respectively, the new objective function in Mayer form reads

$$
J(q, u)=z(\mathrm{~T})+\Phi(q(\mathrm{~T}), \dot{q}(\mathrm{~T}))
$$

resp. $\tilde{J}(q, p, u)=y(\mathrm{~T})+\tilde{\Phi}(q(\mathrm{~T}), p(\mathrm{~T}))$. Equation (2.23) is typically adjoint to the problem description as an additional differential equation of the form $\dot{z}=C(q, \dot{q}, u), z(0)=0$, resp. $\dot{y}=\tilde{C}(q, p, u), y(0)=0$. Note that the Mayer form destroys the unifying Lagrangian and Hamiltonian structures.

Discrete Lagrangian system. As in the continuous setting, the discrete optimal control problem (2.10) can be transformed into a problem in Mayer form, that is the objective function consists of a final condition only. The transformation is performed on the discrete level to keep the Lagrangian structure of the original problem. We introduce new variables $z_{0}=0, z_{\ell}=\sum_{k=0}^{\ell-1} C_{d}\left(q_{k}, q_{k+1}, u_{k}\right), \ell=1, \ldots, N$, and rephrase the discrete problem (2.10) into a problem of Mayer type:

$$
\begin{gathered}
\min _{q_{d}, u_{d}} z_{N}+\Phi_{d}\left(q_{N-1}, q_{N}, u_{N-1}\right) \\
\text { subject to }(2.10 \mathrm{~b})-(2.10 \mathrm{f}) \text { and } z_{0}=0, z_{k+1}-z_{k}-C_{d}\left(q_{k}, q_{k+1}, u_{k}\right)=0, k=0, \ldots, N-1 .
\end{gathered}
$$

Thus equations (2.24b) provide the corresponding discretization for the additional equation of motion resulting from the Mayer transformation of the Lagrangian optimal control problem on the continuous level.

Discrete Hamiltonian system. Similarly, (2.22) can be transformed into an optimal control problem of Mayer type as follows: analogous to what we did in equation (2.24), we introduce additional variables $y_{d}=\left\{y_{l}\right\}_{l=0}^{N}$ as

$$
y_{0}=0, \quad y_{l}=\sum_{k=0}^{l-1} h \sum_{i=1}^{s} b_{i} \tilde{C}\left(Q_{k i}, P_{k i}, U_{k i}\right), \quad l=1, \ldots, N,
$$

yielding the discrete optimal control problem of Mayer type as

$$
\min _{q_{d}, p_{d}, u_{d}} \bar{\Phi}\left(q_{N}, p_{N}, y_{N}\right)=y_{N}+\tilde{\Phi}\left(q_{N}, p_{N}\right)
$$

subject to the constraints in problem (2.22). We obtain exactly the same problem by discretizing the continuous Hamiltonian optimal control problem of Mayer type with the same partitioned Runge-Kutta discretization 
for the extended system of differential equations

$$
\begin{array}{ll}
\dot{\tilde{q}}=\nu(\tilde{q}, p, u), & \tilde{q}(0)=\tilde{q}^{0}, \\
\dot{\tilde{p}}=\eta(\tilde{q}, p, u), & p(0)=p^{0},
\end{array}
$$

with $\tilde{q}=(q, y), \nu(\tilde{q}, p, u)=\left(\nabla_{p} H(q, p), \tilde{C}(q, p, u)\right), \eta(\tilde{q}, p, u)=-\nabla_{p} H(q, p)+f_{H}(q, p, u), \tilde{q}^{0}=\left(q^{0}, 0\right)$.

\subsection{The adjoint systems}

The adjoint system provides necessary optimality conditions for a given optimal control problem. In the continuous case the adjoint system is derived via the Pontryagin maximum principle. The KKT equations provide the adjoint system for the discrete optimal control problem. In [28], a transformed adjoint system is derived which uses standard Runge-Kutta discretizations. Also, order conditions on the coefficients of the adjoint scheme are identified up to order 4. In [9], these are extended up to order 7.

By using the same strategy as Hager in [28], we here show that DMOC leads to a discretization of the same order for the adjoint system as for the state system. Therefore no additional order conditions on the coefficients are necessary. In the following we ignore path and control constraints and restrict ourselves to the case of unconstrained optimal control problems.

Continuous setting. We consider a Hamiltonian optimal control problem in Mayer form without final constraint, path and control constraints, i.e.

\section{Problem 2.12.}

$$
\min _{q, p, u} \Phi(q(\mathrm{~T}), p(\mathrm{~T}))
$$

subject to $\dot{q}=\nu(q, p), \dot{p}=\eta(q, p, u),(q(0), p(0))=\left(q^{0}, p^{0}\right)$, with $q, p \in \mathrm{W}^{1, \infty}\left([0, \mathrm{~T}], \mathbb{R}^{n}\right), u \in \mathrm{L}^{\infty}\left([0, \mathrm{~T}], \mathbb{R}^{m}\right)$, $\nu(q, p)=\nabla_{p} H(q, p), \eta(q, p, u)=-\nabla_{q} H(q, p)+f_{H}(q, p, u)$. We also write $x=(q, p), x^{0}=\left(q^{0}, p^{0}\right)$ and $\tilde{f}(x, u)=$ $(\nu(q, p), \eta(q, p, u))$, such that the problem reads as $\dot{x}=\tilde{f}(x, u), x(0)=x^{0}$.

Along the lines of [28], we now formulate the assumptions that are employed in the analysis of DMOC discretizations of Problem 2.12. First, a smoothness assumptions is required to ensure regularity of the solution and the problem functions. Second, we enforce a growth condition that allows for having a unique solution for the control function of the optimal control problem.

Assumption 2.13 (smoothness). For some integer $\kappa \geq 2$, Problem 2.12 has a local solution $\left(x^{*}, u^{*}\right)$ which lies in $\mathrm{W}^{\kappa, \infty} \times \mathrm{W}^{\kappa-1, \infty}$. There exists an open set $\Omega \subset \mathbb{R}^{2 n} \times \mathbb{R}^{m}$ and $\rho>0$ such that ${ }^{8} B_{\rho}\left(x^{*}(t), u^{*}(t)\right) \subset \Omega$ for every $t \in[0, T]$. The first $\kappa$ derivatives of $\nu$ and $\eta$ are Lipschitz continuous in $\Omega$, and the first $\kappa$ derivatives of $\Phi$ are Lipschitz continuous in $B_{\rho}\left(x^{*}(T)\right)$.

First order optimality conditions. Under Assumption 2.13 there exists an associated Lagrange multiplier $\psi^{*}=\left(\psi^{q, *}, \psi^{p, *}\right) \in W^{\kappa, \infty}$ for which the following form of the first-order optimality conditions derived via the Pontryagin maximum principle is satisfied at $\left(x^{*}, \psi^{*}, u^{*}\right)$ :

$$
\begin{aligned}
& \dot{q}=\nu(q, p), \quad q(0)=q^{0}, \\
& \dot{p}=\eta(q, p, u), \quad p(0)=p^{0}, \\
& \dot{\psi}^{q}=-\nabla_{q} \mathcal{H}\left(q, p, \psi^{q}, \psi^{p}, u\right), \quad \psi^{q}(\mathrm{~T})=\nabla_{q} \Phi(q(\mathrm{~T}), p(\mathrm{~T})) \\
& \dot{\psi}^{p}=-\nabla_{p} \mathcal{H}\left(q, p, \psi^{q}, \psi^{p}, u\right), \quad \psi^{p}(\mathrm{~T})=\nabla_{p} \Phi(q(\mathrm{~T}), p(\mathrm{~T})) \\
& \nabla_{u} \mathcal{H}\left(q(t), p(t), \psi^{q}(t), \psi^{p}(t), u(t)\right)=0 \text { for all } t \in[0, \mathrm{~T}],
\end{aligned}
$$

\footnotetext{
${ }^{8} B_{\rho}(z)$ is the closed ball centered at $z$ with radius $\rho$.
} 
with the Hamiltonian $\mathcal{H}$ defined by $\mathcal{H}\left(q, p, \psi^{q}, \psi^{p}, u\right)=\psi^{q} \nu(q, p)+\psi^{p}$. We also write $\mathcal{H}(x, \psi, u):=\psi \tilde{f}(x, u)$ such that $(2.28 \mathrm{c})$ and $(2.28 \mathrm{~d})$ read as $\dot{\psi}=-\nabla_{x} \mathcal{H}(x, \psi, u)$ with $\psi(\mathrm{T})=\nabla_{x} \Phi(x(\mathrm{~T}))$.

Second order optimality conditions. In order to formulate the second-order sufficient optimality conditions we define the following matrices ( $c f .[28])$ :

$$
\begin{gathered}
A(t)=\nabla_{x} \tilde{f}\left(x^{*}(t), u^{*}(t)\right), B(t)=\nabla_{u} \tilde{f}\left(x^{*}(t), u^{*}(t)\right), V(t)=\nabla^{2} \Phi\left(x^{*}(\mathrm{~T})\right), \\
P(t)=\nabla_{x x} \mathcal{H}\left(x^{*}(t), \psi^{*}(t), u^{*}(t)\right), R(t)=\nabla_{u u} \mathcal{H}\left(x^{*}(t), \psi^{*}(t), u^{*}(t)\right), S(t)=\nabla_{x u} \mathcal{H}\left(x^{*}(t), \psi^{*}(t), u^{*}(t)\right) .
\end{gathered}
$$

Let $\mathcal{B}$ be the quadratic form $\mathcal{B}(x, u)=\frac{1}{2}\left(x(\mathrm{~T})^{T} V x(\mathrm{~T})+\langle x, P x\rangle+\langle u, R u\rangle+2\langle x, S u\rangle\right)$, where $\langle\cdot, \cdot\rangle$ denotes the usual $L^{2}$ inner product over the time interval $[0, \mathrm{~T}]$.

Assumption 2.14 (coercivity). There exists a constant $\alpha>0$ such that $\mathcal{B}(x, u) \geq \alpha\|u\|_{L_{2}}^{2}$ for all $(x, u) \in \mathcal{M}$, where $\mathcal{M}=\left\{(x, u) \in H^{1} \times L^{2} \mid x(0)=0\right.$ and $\dot{x}=A x+B u$ for a.e. $\left.t \in[0, T]\right\}$.

Under the assumptions Smoothness and Coercivity, the control uniqueness property holds (cf. [27]): the Hamiltonian $\mathcal{H}$ has a locally unique minimizer $u^{*}=\hat{u}(x, \psi)$ in the control, depending Lipschitz continuously on $x$ and $\psi$ with $\hat{u}: T^{*} Q \times \mathbb{R}^{2 n} \rightarrow U$. Let $\phi=\left(\phi^{q}, \phi^{p}\right)$ denote the function defined by $\phi^{q}(x, \psi)=-\nabla_{q} \mathcal{H}(x, \psi, \hat{u}(x, \psi))$, $\phi^{p}(x, \psi)=-\nabla_{p} \mathcal{H}(x, \psi, \hat{u}(x, \psi))$. Additionally, let $\eta(x, \psi)=\eta\left(x, u^{*}\right)$. By substituting $u^{*}=\hat{u}(x, \psi)$ into $(2.28)$ one obtains a two-point boundary-value problem with

$$
\dot{\psi}^{q}=\phi^{q}\left(q, p, \psi^{q}, \psi^{p}\right), \quad \dot{\psi}^{p}=\phi^{p}\left(q, p, \psi^{q}, \psi^{p}\right) .
$$

Note that the functions $\phi^{q}$ and $\phi^{p}$ are defined only implicitly. These can be solved for during simulation.

Discrete setting. It was shown in Section 2.4 that the discrete Lagrangian control problem is equivalent to problem (2.22) (resp. (2.26)). Using some transformation and change of variables (see [28,71]), by reversing the order of time and applying the control uniqueness property, we obtain the following version of the first-order necessary optimality conditions associated with (2.22) (the Karush-Kuhn-Tucker conditions):

$$
\begin{aligned}
q_{k+1} & =q_{k}+h \sum_{i=1}^{s} b_{i} \nu\left(Q_{k i}, P_{k i}\right), \quad q_{0}=q^{0}, \\
p_{k+1} & =p_{k}+h \sum_{i=1}^{s} b_{i} \eta\left(Q_{k i}, P_{k i}, \Psi_{k i}^{q}, \Psi_{k i}^{p}\right), \quad p_{0}=p^{0}, \\
Q_{k i} & =q_{k}+h \sum_{j=1}^{s} a_{i j}^{q} \nu\left(Q_{k j}, P_{k j}\right), \quad P_{k i}=p_{k}+h \sum_{j=1}^{s} a_{i j}^{p} \eta\left(Q_{k j}, P_{k j}, \Psi_{k j}^{q}, \Psi_{k j}^{p}\right), \\
\psi_{k+1}^{q} & =\psi_{k}^{q}+h \sum_{i=1}^{s} b_{i} \phi^{q}\left(Q_{k i}, P_{k i}, \Psi_{k i}^{q}, \Psi_{k i}^{p}\right), \quad \psi_{N}^{q}=\nabla_{q} \Phi\left(q_{N}, p_{N}\right), \\
\psi_{k+1}^{p} & =\psi_{k}^{p}+h \sum_{i=1}^{s} b_{i} \phi^{p}\left(Q_{k i}, P_{k i}, \Psi_{k i}^{q}, \Psi_{k i}^{p}\right), \quad \psi_{N}^{p}=\nabla_{p} \Phi\left(q_{N}, p_{N}\right), \\
\Psi_{k i}^{q} & =\psi_{k}^{q}+h \sum_{j=1}^{s} \bar{a}_{i j}^{q} \phi^{q}\left(Q_{k j}, P_{k j}, \Psi_{k j}^{q}, \Psi_{k j}^{p}\right), \quad \Psi_{k i}^{p}=\psi_{k}^{p}+h \sum_{j=1}^{s} \bar{a}_{i j}^{p} \phi^{p}\left(Q_{k j}, P_{k j}, \Psi_{k j}^{q}, \Psi_{k j}^{p}\right), \\
\bar{a}_{i j}^{q} & =\frac{b_{i} b_{j}-b_{j} a_{j i}^{q}}{b_{i}}, \quad \bar{a}_{i j}^{p}=\frac{b_{i} b_{j}-b_{j} a_{j i}^{p} .}{b_{i}} .
\end{aligned}
$$

Assuming $h$ small enough and $x_{k}=\left(q_{k}, p_{k}\right)$ near $x^{*}\left(t_{k}\right)=\left(q^{*}\left(t_{k}\right), p^{*}\left(t_{k}\right)\right)$ the intermediate states $Q_{k i}$ and $P_{k i}$ and costates $\Psi_{k i}^{q}$ and $\Psi_{k i}^{p}$ are uniquely determined since $\hat{u}(x, \psi)$ depends Lipschitz continuously on $x$ near $x^{*}(t)$ and $\psi$ near $\psi^{*}(t)$ for any $t \in[0, \mathrm{~T}]$. This follows from smoothness and the implicit function theorem 
as for standard Runge-Kutta discretization as stated in [28] (see for example [23]). Thus, there exists a locally unique solution $\left(Q_{k i}, P_{k i}, \Psi_{k i}^{q}, \Psi_{k i}^{p}\right), 1 \leq i \leq s$ of (2.30d)-(2.30f).

The scheme (2.30) can be viewed as a discretization of the two-point boundary-value problem (2.28), (2.29). To ensure a desired order of approximation for this two-point boundary-value problem, [28] derives order conditions on the coefficients of the Runge-Kutta scheme via Taylor expansions. In our case, however, we more easily obtain the following result concerning the order of consistency.

Theorem 2.15 (order of consistency). If the symplectic partitioned Runge-Kutta discretization of the state system is of order $\kappa$ and $b_{i}>0$ for each $i$, then the scheme for the adjoint system is again a symplectic partitioned Runge-Kutta scheme of the same order (in particular, both schemes are the same).

Proof. Starting with a symplectic partitioned Runge-Kutta discretization for the state system (2.27), the discrete necessary optimality conditions are given as the adjoint system $(2.30 \mathrm{~d})-(2.30 \mathrm{~g})$. This system is again a partitioned Runge-Kutta scheme for the adjoint equations with coefficients $b_{i}, \bar{a}_{i j}^{q}, \bar{a}_{i j}^{p}$. Substituting the symplecticity condition $a_{i j}^{p}=\frac{b_{i} b_{j}-b_{j} a_{j i}^{q}}{b_{i}}$ into equation $(2.30 \mathrm{~g})$, the coefficients are determined as $\bar{a}_{i j}^{q}=a_{i j}^{p}$, and $\bar{a}_{i j}^{p}=a_{i j}^{q}$. Since the coefficients of the Runge-Kutta scheme of the adjoint system for $\left(\psi^{p}, \psi^{q}\right)$ are the same as the coefficients of the Runge-Kutta scheme of the state system for $(q, p)$ the adjoint scheme is of same order.

With Theorems 1.16 and 2.8 one obtains the following:

Lemma 2.16. Let a Lagrangian optimal control problem with regular Lagrangian $L$ and Lagrangian control force $f_{L}$ be given. If the discrete Lagrangian $L_{d}(2.20)$ and the discrete control force $f_{d}^{ \pm}(2.21)$ with $b_{i}>0$ for each $i$ are both of order $\kappa$, then the corresponding adjoint scheme is also of order $\kappa$.

\subsection{Convergence}

The purpose of this section is to establish the convergence of solutions of the discrete optimal control problem (2.10) to a solution of the Lagrangian optimal control problem (2.3) as the step size $h \rightarrow 0$. As in Section 2.6, we restrict ourselves to the case without path and control constraints and without final point constraint. Our convergence statement is a direct application of the one in [21] (cf. also [28,71]). We need the following abstract result:

Theorem 2.17 ([29]). Let $\mathcal{X}$ be a Banach space and let $\mathcal{Y}$ be a linear normed space with the norm in both spaces denoted by $\|\cdot\|$. Let $\mathcal{F}: \mathcal{X} \mapsto 2^{\mathcal{Y}}$ be a set-valued map, let $\mathcal{L}: \mathcal{X} \mapsto \mathcal{Y}$ be a bounded, linear operator, and let $\mathcal{T}: \mathcal{X} \mapsto \mathcal{Y}$ with $\mathcal{T}$ continuously Frechét differentiable in $B_{r}\left(w^{*}\right)$ for some $w^{*} \in \mathcal{X}$ and $r>0$. Suppose that the following conditions hold for some $\delta \in \mathcal{Y}$ and scalars $\epsilon, \lambda$ and $\sigma>0$ :

(P1) $\mathcal{T}\left(w^{*}\right)+\delta \in \mathcal{F}\left(w^{*}\right)$;

(P2) $\|D \mathcal{T}(w)-\mathcal{L}\| \leq \epsilon$ for all $w \in B_{r}\left(w^{*}\right)$;

(P3) the map $(\mathcal{F}-\mathcal{L})^{-1}$ is single-valued and Lipschitz continuous in $B_{\sigma}(\pi), \pi=(\mathcal{T}-\mathcal{L})\left(w^{*}\right)$ with Lipschitz constant $\lambda$.

If $\epsilon \lambda<1, \epsilon r \leq \sigma$, and $\|\delta\| \leq(1-\lambda \epsilon) r / \lambda$, then there exists a unique $w \in B_{r}\left(w^{*}\right)$ such that $\mathcal{T}(w) \in \mathcal{F}(w)$. Moreover, we have the estimate

$$
\left\|w-w^{*}\right\| \leq \frac{\lambda}{1-\lambda \epsilon}\|\delta\| .
$$

Consistency corresponds to assumption (P1) and the bounds on the norm of $\delta$, stability corresponds to assumption (P3) and the bound on the Lipschitz constant $\lambda$ for the linearization, and convergence is stated in (2.31). The following convergence result is formulated in terms of the averaged modulus of smoothness of the optimal control. If $J \subset \mathbb{R}$ is an interval and $v: J \rightarrow \mathbb{R}^{n}$, let $\omega(v, J ; t, h)$ denote the modulus of continuity: $\omega(v, J ; t, h)=\sup \left\{\left|v\left(s_{1}\right)-v\left(s_{2}\right)\right|: s_{1}, s_{2} \in[t-h / 2, t+h / 2] \cap J\right\}$. The averaged modulus of smoothness $\tau$ of $v$ over $[0, \mathrm{~T}]$ is the integral of the modulus of continuity: $\tau(v ; h)=\int_{0}^{\mathrm{T}} \omega(v,[0, \mathrm{~T}] ; t, h) \mathrm{d} t$. It is shown in [79] that $\lim _{h \rightarrow 0} \tau(v ; h)=0$ if and only if $v$ is Riemann integrable, and $\tau(v ; h) \leq c h$ with constant $c$ if $v$ has bounded variation. 
Theorem 2.18 (convergence). Let $\left(x^{*}, u^{*}\right)$ be a solution of the Lagrangian optimal control problem (2.3a)(2.3b). If smoothness and coercivity hold, the discrete Lagrangian $L_{d}(2.20)$ and the discrete control force $f_{d}^{ \pm}$ (2.21) are of order $\kappa$ with $b_{i}>0$ for each $i$, then for all sufficiently small $h$ there exists a strict local minimizer $\left(x^{h}, u^{h}\right)$ of the discrete Lagrangian optimal control problem (2.10) and an associated adjoint variable $\psi^{h}$ satisfying the first-order necessary optimality conditions such that

$$
\max _{0 \leq k \leq N}\left|x_{k}^{h}-x^{*}\left(t_{k}\right)\right|+\left|\psi_{k}^{h}-\psi^{*}\left(t_{k}\right)\right|+\left|u\left(x_{k}^{h}, \psi_{k}^{h}\right)-u^{*}\left(t_{k}\right)\right| \leq c h^{\kappa-1}\left(h+\tau\left(\partial_{t}^{\kappa-1} u^{*} ; h\right)\right),
$$

where $u\left(x_{k}^{h}, \psi_{k}^{h}\right)$ is a local minimizer of the Hamiltonian $\mathcal{H}$ corresponding to $x=x_{k}$ and $\psi=\psi_{k}$.

Proof. With (1.16) and by Theorem 2.8 we know that a discrete Lagrangian and discrete forces both of order $\kappa$ lead to a symplectic partitioned Runge-Kutta discretization of order $\kappa$ for the state system. Because of smoothness and coercivity we can build up a discrete adjoint scheme with eliminated control that approximates the continuous adjoint scheme with order $\kappa$ (see Lem. 2.16). This leads in Hager's terminology to a RungeKutta scheme of order $\kappa$ for optimal control, and therefore Hager's convergence result for standard Runge-Kutta schemes in [28], Theorem 2.1, is directly applicable.

Remark 2.19. The estimate for the error in the discrete control in (2.32) is expressed in terms of $\hat{u}\left(x_{k}^{h}, \psi_{k}^{h}\right)$ not $u_{k}$. This is due to the fact, that we derive the estimate via the transformed adjoint system with removed control due to the control uniqueness property. In [21] the estimate is proved in terms of $u_{k}$ for Runge-Kutta discretization of second order.

Remark 2.20. Theorem 2.18 can be extended to optimal control problems with constraints on the control function $u(t)$ as it was done in [28] for Runge-Kutta discretizations of order 2.

\section{IMPLEMENTATION AND APPLICATIONS}

\subsection{Implementation}

As a balance between accuracy and efficiency we employ the midpoint rule for approximating the relevant integrals for the example computations in the following section, that is we set

$$
\begin{aligned}
& C_{d}\left(q_{k}, q_{k+1}, u_{k}\right)=h C\left(\frac{q_{k+1}+q_{k}}{2}, \frac{q_{k+1}-q_{k}}{h}, u_{k+1 / 2}\right), \quad L_{d}\left(q_{k}, q_{k+1}\right)=h L\left(\frac{q_{k+1}+q_{k}}{2}, \frac{q_{k+1}-q_{k}}{h}\right), \\
& \int_{k h}^{(k+1) h} f_{L}(q(t), \dot{q}(t), u(t)) \cdot \delta q(t) \mathrm{d} t \approx h f_{L}\left(\frac{q_{k+1}+q_{k}}{2}, \frac{q_{k+1}-q_{k}}{h}, u_{k+1 / 2}\right) \cdot \frac{\delta q_{k+1}+\delta q_{k}}{2}, k=0, \ldots, N-1 .
\end{aligned}
$$

Here, $f_{k}^{-}=f_{k}^{+}=\frac{h}{2} f_{L}\left(\frac{q_{k+1}+q_{k}}{2}, \frac{q_{k+1}-q_{k}}{h}, u_{k+1 / 2}\right)$ are used as the left and right discrete forces with $q_{k}=q\left(t_{k}\right)$ and $u_{k+1 / 2}=u\left(\frac{t_{k}+t_{k+1}}{2}\right)$.

SQP method. We solve the resulting finite dimensional constrained optimization problem by a standard SQP method as implemented for example in the routine fmincon of MATLAB. For more complex problems we use the routine nag_opt_nlp_sparse of the NAG library ${ }^{9}$. Since SQP is a local method, different initial guesses can lead to different solutions. This has been observed in almost all our example computations.

Automatic differentiation. The SQP method makes use of the first and second derivatives of the constraints and the objective function. In the case where no derivatives are provided, the code approximates those by finite differences. This approximation is time-consuming and cancellation errors lead to bad convergence behavior. In order to avoid these drawbacks we make use of the concept of Automatic Differentiation (AD) (see [26,77,87]), a method to numerically evaluate the derivative of a function specified by a computer program. In our implementation we used the package ADOL-C (Automatic Differentiation by OverLoading in $\mathbf{C}++[84]$ ).

\footnotetext{
9 wWw. nag. com.
} 


\subsection{Applications}

In this section, we numerically compare DMOC to a collocation method by means of two problems: a low thrust orbital transfer and the optimal control of a two-link manipulator. For this comparison, we apply a collocation method of order 2 to two different models: the Hamiltonian system with coordinates $(q, p)$ as well as the system formulated on tangent bundle with coordinates $(q, \dot{q})=(q, v)$.

\subsubsection{Low thrust orbital transfer}

Model. Consider a satellite with mass $m$ which moves in the gravitational field of the Earth (mass $M$ ). The satellite is to be transferred from one circular orbit to one in the same plane with a larger radius, while the number of revolutions around the Earth during the transfer process is fixed. In 2d-polar coordinates $q=(r, \varphi)$, the Lagrangian of the system has the form

$$
L(q, \dot{q})=\frac{1}{2} m\left(\dot{r}^{2}+r^{2} \dot{\varphi}^{2}\right)+\gamma \frac{M m}{r}
$$

where $\gamma$ denotes the gravitational constant. Assume that the propulsion system continuously exhibits a force $u$ only in the direction of circular motion (i.e. orthogonal to the vector $r$ ), such that the corresponding Lagrangian control force is given by $f_{L}=(0, r u)^{T}$.

Boundary conditions. Assume further that the satellite initially moves on a circular orbit of radius $r^{0}$. Let $(r(0), \varphi(0))=\left(r^{0}, 0\right)$ be its position at $t=0$, then its initial velocity is given by $\dot{r}(0)=0$ and $\dot{\varphi}(0)=\sqrt{\gamma M /\left(r^{0}\right)^{3}}$. Using its thruster, the satellite is required to reach the point $\left(r^{\mathrm{T}}, 0\right)$ at time $\mathrm{T}=d \sqrt{\frac{4 \pi^{2}}{8 \gamma M}\left(r^{0}+r^{\mathrm{T}}\right)^{3}}$ and, without any further control input, to continue to move on the circle with radius $r^{\mathrm{T}}$. Here, $d$ is a prescribed number of revolutions around the Earth. Thus, the boundary values at $t=\mathrm{T}$ are given by $(r(\mathrm{~T}), \varphi(\mathrm{T}))=\left(r^{\mathrm{T}}, 0\right)$ and $(\dot{r}(\mathrm{~T}), \dot{\varphi}(\mathrm{T}))=\left(0, \sqrt{\gamma M /\left(r^{\mathrm{T}}\right)^{3}}\right)$.

Objective functional. During this transfer, our goal is to minimize the control effort, correspondingly the objective functional is given by

$$
J(u)=\int_{0}^{\mathrm{T}} u(t)^{2} \mathrm{~d} t .
$$

Results. We compute the transfer from an orbit of radius $r^{0}=30 \mathrm{~km}$ to one of radius $r^{\mathrm{T}}=330 \mathrm{~km}$ around the Earth. The optimal control for a transfer within one revolution $(d=1)$ around the Earth is shown in Figure 3a. First, we investigate how well the balance between the change in angular momentum and the amount of the control force is preserved. Due to the invariance of the Lagrangian under the rotation $\varphi$, the angular momentum of the satellite is preserved in the absence of external forces (as stated in Noether's theorem). However, in the presence of control forces, equation (1.5) gives a relation between the forces and the evolution of the angular momentum.

In Figure 3b, we compare the amount of the acting force with the change in angular momentum in each time interval. For the solution resulting from DMOC and the collocation approach applied to the Hamiltonian system, the change in angular momentum exactly equals the sum of the applied control forces (to numerical accuracy). The collocation method of second order corresponds to a discretization via the implicit midpoint rule. Thus, the optimization problem resulting from DMOC is equivalent to that obtained by applying collocation to the Hamiltonian formulation of the system as shown in Theorem 2.9 and Example 2.10a. Obviously, we obtain equal solutions by applying both methods. These results are consistent with the well-known conservation properties of variational integrators, that provide discretizations that preserve the continuous properties as momentum maps in the discrete setting in a natural way. On the other hand, the collocation method applied to the tangent space system described in velocities fails to capture the change in angular momentum accurately because the discrete tangent space formulation destroys the discrete Hamiltonian structure and the resulting (also unforced) scheme is not momentum-preserving anymore. 


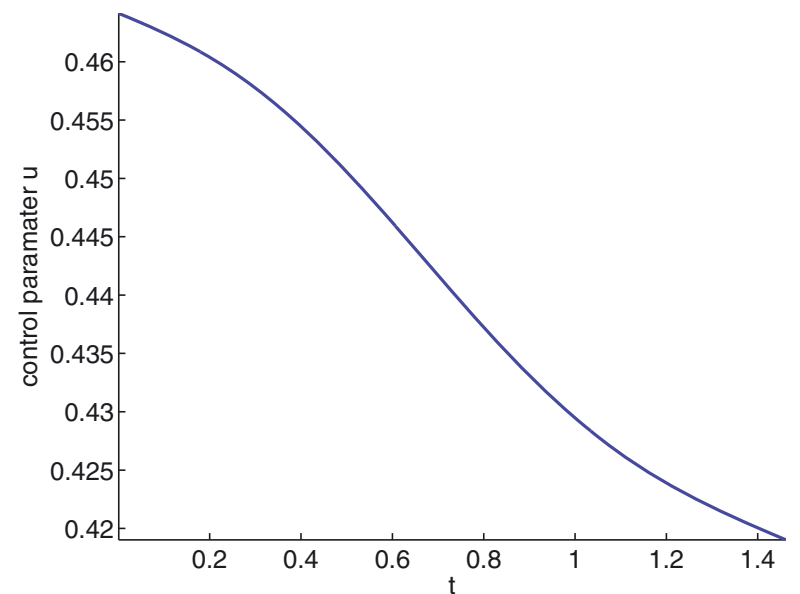

a)

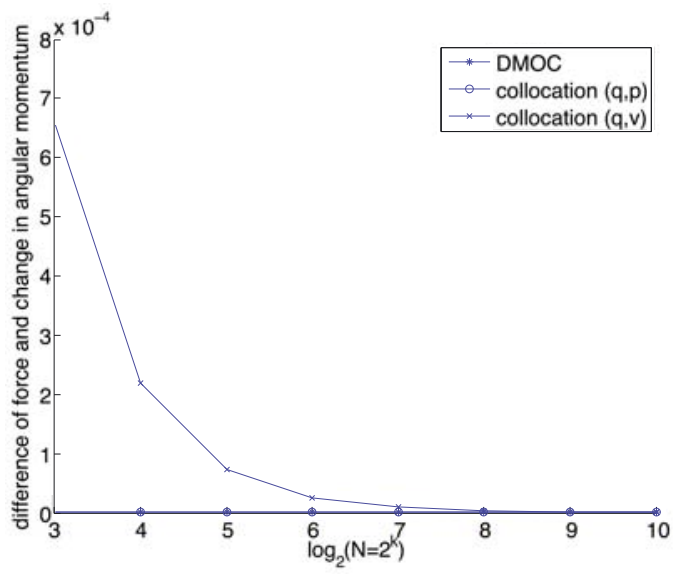

b)

Figure 3. (a) Optimal control trajectory for a satellite transfer. (b) Comparison of the accuracy of the computed open loop control for DMOC and a collocation approach: difference of force and change in angular momentum in dependence on the number of discretization points. The lines for DMOC and collocation $(q, p)$ lie on top of each other.

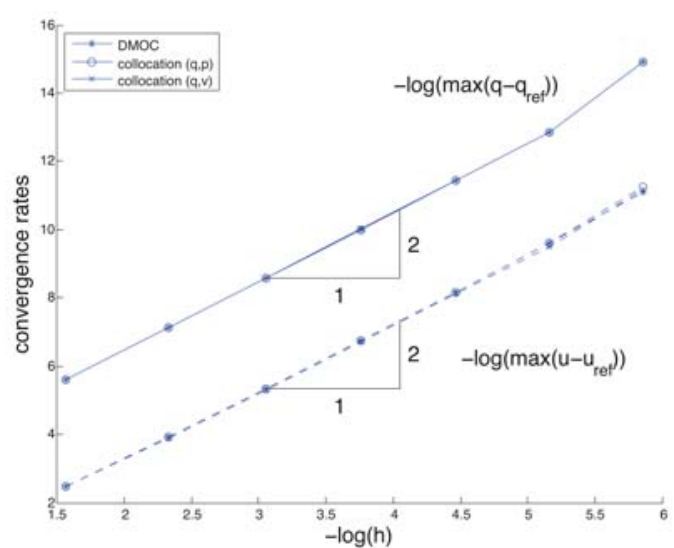

a)

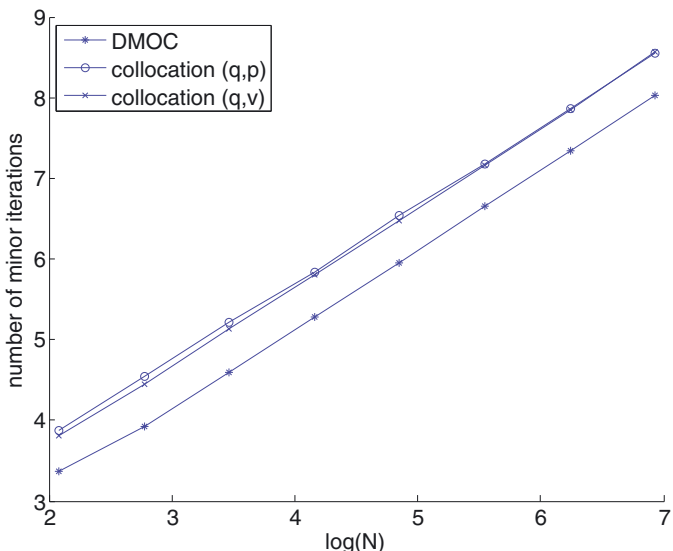

b)

Figure 4. (a) Approximation error for the configurations (solid) and the controls (dashed) in dependence of the step size for DMOC and a collocation approach. For all three methods the lines lie almost on top of each other. (b) Comparison of the number of iteration steps performed by the SQP solver for DMOC and a collocation approach in dependence on the number of discretization points.

In Figure 4a we show the convergence rates for all three methods. A reference trajectory is computed with $N=1024$ discretizations points and time step $h=2.9 \times 10^{-3}$. The error in the configuration and control parameter of the discrete solution with respect to the reference solution is computed as $\max _{k=0, \ldots, N} \mid q\left(t_{k}\right)-$ $q_{\text {ref }}\left(t_{k}\right) \mid$ and $\max _{k=0, \ldots, N}\left|u\left(t_{k}\right)-u_{\text {ref }}\left(t_{k}\right)\right|$, respectively, where $|\cdot|$ is the Euclidean norm. For all three methods the convergence rate for the configuration and control trajectory is $\mathcal{O}\left(h^{2}\right)$, as predicted by Theorem 2.18.

Still, DMOC is advantageous regarding the computational efficiency. Due to its formulation on the discrete configuration space, DMOC only uses $\frac{3}{5} \approx 0.6$ of the number of variables of the collocation approach 


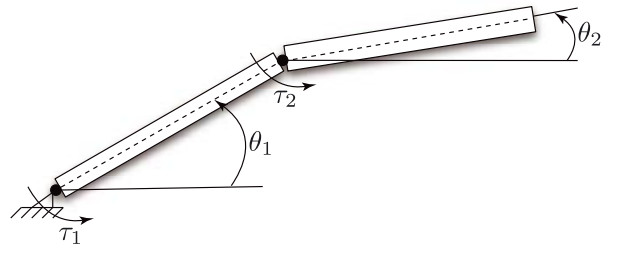

a)

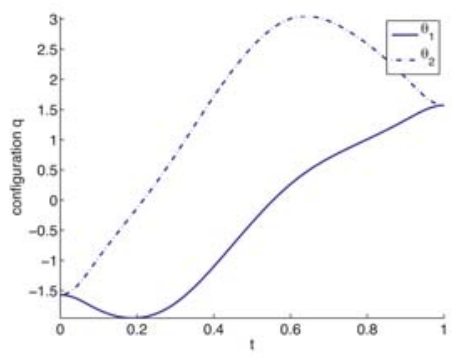

b)

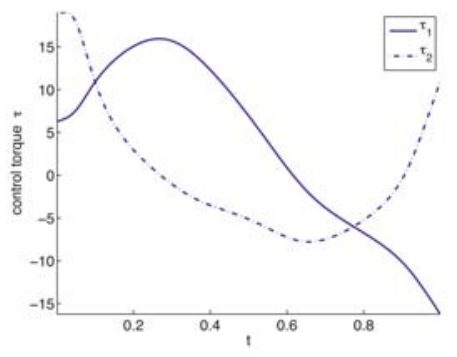

c)

Figure 5. (a) Model of a two-link manipulator. (b) Optimal configuration trajectories.

(c) Optimal control trajectories.

(cf. Rem. 2.11). Figure 4b shows the number of all iterations that the SQP solver performs in order to solve the quadratic subproblems (minor iterations). We observe that for DMOC the SQP solver needs about 1.7 times less iterations in comparison to collocation. This is in exact agreement with the reduced number of variables.

\subsubsection{Two-link manipulator}

As a second numerical example we consider the optimal control of a two-link manipulator. Again, we compare DMOC to a collocation method of the same order.

Model. The two-link manipulator (see Fig. 5a) consists of two coupled planar rigid bodies with mass $m_{i}$, length $l_{i}$ and moment of inertia $J_{i}, i=1,2$, respectively. For $i \in 1,2$, we let $\theta_{i}$ denote the orientation of the $i$ th link measured counterclockwise from the positive horizontal axis. If we assume one end of the first link to be fixed in an inertial reference frame, the configuration of the system is specified by $q=\left(\theta_{1}, \theta_{2}\right)$.

The Lagrangian is given via the kinetic and potential energy

$$
K(q, \dot{q})=\frac{1}{8}\left(m_{1}+4 m_{2}\right) l_{1}^{2} \dot{\theta}_{1}^{2}+\frac{1}{8} m_{2} l_{2}^{2} \dot{\theta}_{2}^{2}+\frac{1}{2} m_{2} l_{1} l_{2} \cos \left(\theta_{1}-\theta_{2}\right) \dot{\theta}_{1} \dot{\theta}_{2}+\frac{1}{2} J_{1} \dot{\theta}_{1}^{2}+\frac{1}{2} J_{2} \dot{\theta}_{2}^{2}
$$

and $V(q)=\frac{1}{2} m_{1} g l_{1} \sin \theta_{1}+m_{2} g l_{1} \sin \theta_{1}+\frac{1}{2} m_{2} g l_{2} \theta_{2}$, with the gravitational acceleration $g$. Control torques $\tau_{1}, \tau_{2}$ are applied at the base of the first link and at the joint between the two links. This leads to the Lagrangian control force $f_{L}\left(\tau_{1}, \tau_{2}\right)=\left(\tau_{1}-\tau_{2}, \tau_{2}\right)^{T}$.

Boundary conditions. The two-link manipulator is to be steered from the stable equilibrium $q^{0}=\left(-\frac{\pi}{2},-\frac{\pi}{2}\right)$ with zero angular velocity $\dot{q}^{0}=(0,0)$ to the unstable equilibrium point $q^{\mathrm{T}}=\left(\frac{\pi}{2}\right.$, $\left.\frac{\pi}{2}\right)$ with velocity $\dot{q}^{\mathrm{T}}=(0,0)$.

Objective functional. Fixing the final time $\mathrm{T}=1$, we would like to minimize the control effort

$$
J\left(\tau_{1}, \tau_{2}\right)=\int_{0}^{\mathrm{T}} \frac{1}{2}\left(\tau_{1}^{2}(t)+\tau_{2}^{2}(t)\right) \mathrm{d} t .
$$

Results. The optimal configuration and control trajectories are shown in Figures 5b and 5c, respectively. In Figure 6 we show (a) the resulting cost and (b) the difference of the amount of force (including the control and the gravitational force) and the change in angular momentum in dependence on the number of discretization points for all three methods. As expected, we obtain (numerically) identical solutions for DMOC and the equivalent collocation method for the Hamiltonian formulation. The midpoint rule applied to the tangent space formulation performs equally well with respect to the objective value evolution. However, as in the previous example it does not reflect the momentum-force consistency as good as the other methods as shown in Figure 6. In Figure 7a the convergence rates are depicted. Here, a reference trajectory has been computed with $N=512$ discretizations points and time step $h=1.9 \times 10^{-3}$. For all three methods the convergence rate for the configuration and control trajectory is $\mathcal{O}\left(h^{2}\right)$, as expected for a scheme of second order accuracy. 


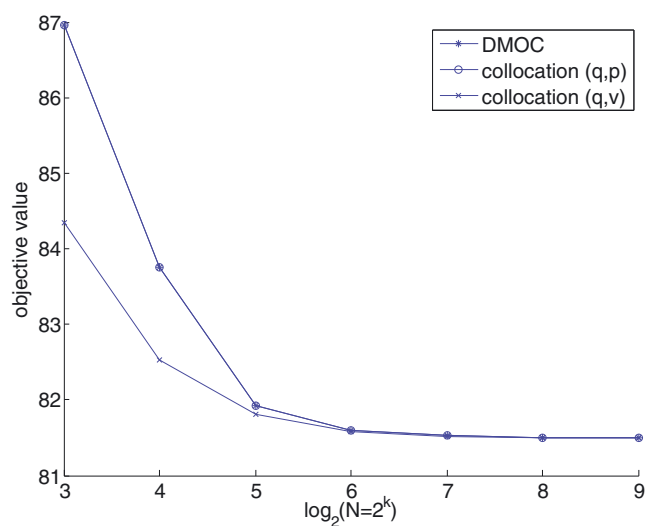

a)

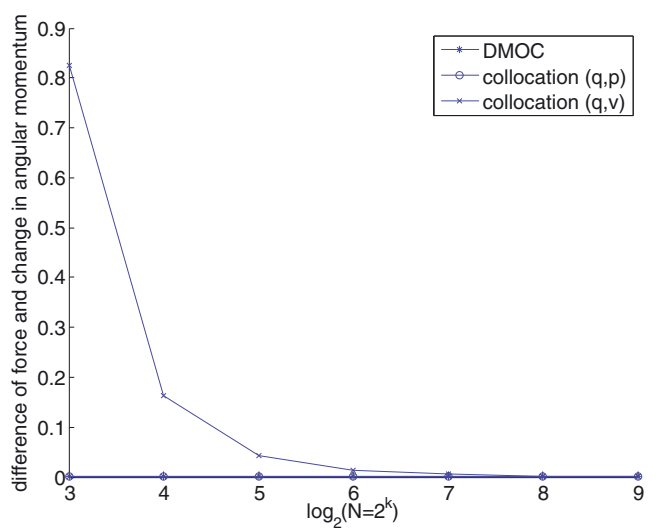

b)

Figure 6. Comparison of the accuracy of the computed open loop control for DMOC and a collocation approach: (a) approximated cost; (b) difference of force and change in angular momentum in dependence on the number of discretization points. The lines for DMOC and collocation $(q, p)$ lie on top of each other.

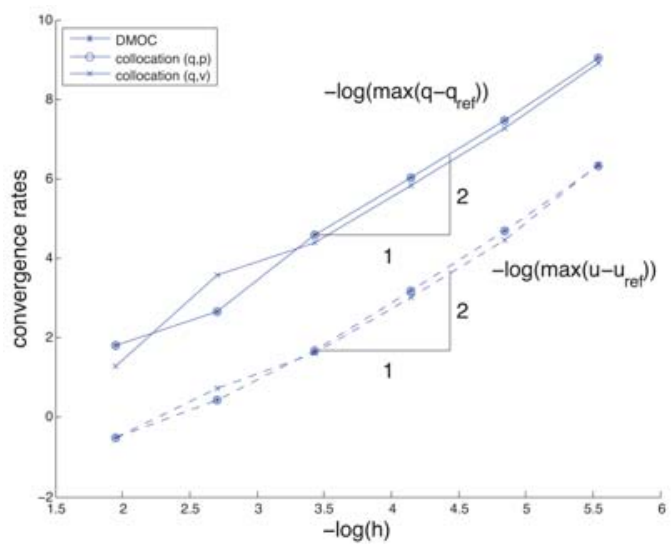

a)

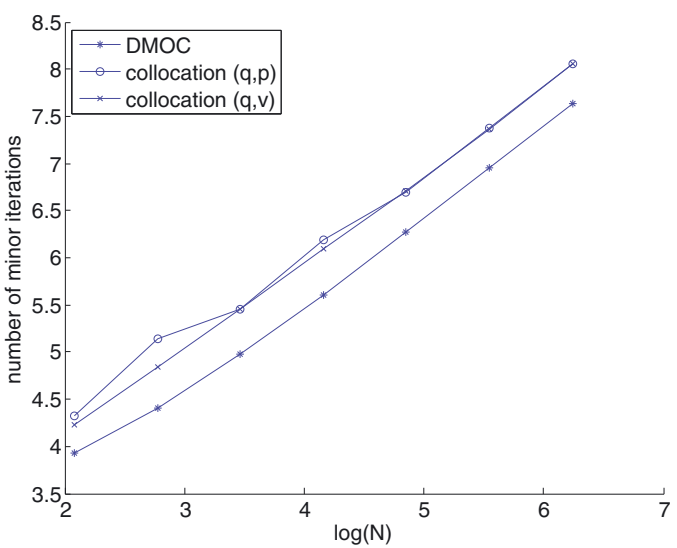

b)

Figure 7. (a) Comparison of the convergence rates for the configurations (solid) and controls (dashed) in dependence of the time step $h$ for DMOC and a collocation approach. The lines for DMOC and collocation $(q, p)$ lie on top of each other. (b) Comparison of the number of iteration steps performed by the SQP solver for DMOC and a collocation approach in dependence on the number of discretization points.

In Figure 7b the number of minor iterations that the SQP solver performs to solve the quadratic subproblems is shown. Similar to what we have seen in the orbital transfer, we observe that the SQP solver needs about 1.5 times less iterations when using DMOC rather than a collocation approach. This factor reflects the fact, that DMOC uses only $\frac{2}{3} \approx 0.67$ of the number of variables in comparison to the collocation approach.

\section{Conclusions And Future Directions}

In this paper we developed a fully discrete formulation of the optimal control of mechanical systems. Based on discrete variational principles, the discrete optimal control problem yields a strategy, denoted by DMOC, 
for the efficient solution of these kinds of problems. The benefits of using DMOC are twofold: On the one hand, in the presence of a symmetry group in the continuous dynamical system, it is known that the momentum map changes in a definite way. The use of discrete mechanics allows one to find an exact counterpart to this on the discrete level. In this paper, this behavior was shown numerically for specific examples. On the other hand, due to the fact that DMOC is implemented on the configuration level rather than on the configuration-momentum or configuration-velocity level, one gets significant computational savings for the number of iterations steps the SQP solver needs to solve the optimization problem. Again, this was demonstrated numerically in examples.

Although the developed method works very successfully in many examples considered in this paper and others (see [35-37,57,71]), there are still some open challenges that will be investigated in future work.

Global minima. One challenge is the issue of local versus global minima. The use of an SQP solver for solving the resulting optimization problem restricts one to local minima that are dependent on the initial guess. One approach to overcome this issue is the use of DMOC primitives $[25,45]$. The authors create a roadmap of feasible trajectories given by a graph where the edges represent small pre-computed DMOC segments referred to as DMOC primitives. A feasible solution of the optimal control problem corresponds to a specific concatenation of the DMOC primitives respecting the dynamics. The global optimal control can be approximated by determining the optimal path in the graph with a given objective function with a global search using techniques from dynamic programming. Initial demonstrations of this idea show that it can be carried out in real time. This, together with the fact that real dynamics is included via DMOC primitives are the significant advantages of this approach.

Adaptive time-stepping. For many applications, an adaptive time-stepping strategy is essential. For example, for problems in space mission design, the planned trajectories require a finer time-stepping nearby planets due to the strong influence of gravity, while for a transfer in nearly free space only few discretization points are necessary to accurately reflect the dynamics of the system. Here, different strategies such as error control based on the discretization grid under consideration and variational approaches could be investigated. For the variational approach a constraint is included to the Lagrange-d'Alembert principle that ensures time step control directly at the level of the discrete action [44]. According to different adaption schemes different constraints can be included such as adaption to acceleration or the strength of control forces.

Convergence involving path constraints. A proof of convergence involving path constraints becomes challenging due to smoothness issues: changes in the active set result into different DAE system which may lead to an order reduction of accuracy and convergence. Only few work has been done and mostly for specific cases (see e.g. $[13,20]$ ) Active set changes are important to investigate and will be subject of future investigations.

Miscellaneous. The framework could be extended to the optimal control of mechanical systems with stochastic influence or contact problems, respectively making use of the theory of stochastic [10] and nonsmooth variational integrators [22], respectively. Due to the variational formulation of DMOC, an extension towards the optimal control of partial differential equations for the treatment of fluid and continuum mechanics might be interesting as well (see $[55,63,65]$ for basic extensions of variational integrators to the context of PDEs).

\section{REFERENCES}

[1] U. Ascher, J. Christiansen and R.D. Russell, A collocation solver for mixed order systems of boundary value problems. Math. Comput. 33 (1979) 659-679.

[2] V. Bär, Ein Kollokationsverfahren zur numerischen Lösung allgemeiner Mehrpunktrandwertaufgaben mit Schalt- und Sprungbedingungen mit Anwendungen in der Optimalen Steuerung und der Parameteridentifizierung. Diploma Thesis, Bonn, Germany (1983).

[3] J.T. Betts, Survey of numerical methods for trajectory optimization. AIAA J. Guid. Control Dyn. 21 (1998) 193-207.

[4] J.T. Betts and W.P. Huffmann, Mesh refinement in direct transcription methods for optimal control. Optim. Control Appl. Meth. 19 (1998) 1-21.

[5] A.I. Bobenko and Y.B. Suris, Discrete Lagrangian reduction, discrete Euler-Poincaré equations, and semidirect products. Lett. Math. Phys. 49 (1999) 79-93.

[6] A.I. Bobenko and Y.B. Suris, Discrete time Lagrangian mechanics on Lie groups, with an application to the Lagrange top. Comm. Math. Phys. 204 (1999) 147-188. 
[7] H.G. Bock, Numerical solutions of nonlinear multipoint boundary value problems with applications to optimal control. Z. Angew. Math. Mech. 58 (1978) T407-T409.

[8] H.G. Bock and K.J. Plitt, A multiple shooting algorithm for direct solution of optimal control problems, in 9th IFAC World Congress, Budapest, Hungary, Pergamon Press (1984) 242-247.

[9] J.F. Bonnans and J. Laurent-Varin, Computation of order conditions for symplectic partitioned Runge-Kutta schemes with application to optimal control. Numer. Math. 103 (2006) 1-10.

[10] N. Bou-Rabee and H. Owhadi, Stochastic variational integrators. IMA J. Numer. Anal. 29 (2008) 421-443.

[11] A.E. Bryson and Y.C. Ho, Applied Optimal Control. Hemisphere (1975).

[12] R. Bulirsch, Die Mehrzielmethode zur numerischen Lösung von nichtlinearen Randwertproblemen und Aufgaben der optimalen Steuerung. Report of the Carl-Cranz-Gesellschaft e.V., DLR, Oberpfaffenhofen, Germany (1971).

[13] C. Büskens and H. Maurer, SQP-methods for solving optimal control problems with control and state constraints: adjoint variables, sensitivity analysis and real-time control. J. Comput. Appl. Math. 120 (2000) 85-108.

[14] J.A. Cadzow, Discrete calculus of variations. Int. J. Control 11 (1970) 393-407.

[15] J.A. Cadzow, Discrete-Time Systems: An Introduction With Interdisciplinary Applications. Prentice-Hall (1973).

[16] A.L. Cauchy, Méthode générale pour la résolution des systèmes d'équations simultanées. C. R. Acad. Sci. 25 (1847) $536-538$.

[17] F.L. Chernousko and A.A. Luybushin, Method of successive approximations for optimal control problems (survey paper). Opt. Control Appl. Meth. 3 (1982) 101-114.

[18] P. Deufhard, A modified Newton method for the solution of ill-conditioned systems of nonlinear equations with application to multiple shooting. Numer. Math. 22 (1974) 289-315.

[19] E.D. Dickmanns and K.H. Well, Approximate solution of optimal control problems using third order hermite polynomial functions. Lect. Notes Comput. Sci. 27 (1975) 158-166.

[20] A.L. Dontchev and W.W. Hager, The Euler Approximation in State Constrained Optimal Control, in Mathematics of Computation 70, American Mathematical Society, USA (2001) 173-203.

[21] A.L. Dontchev, W.W. Hager and V.M. Veliov, Second order Runge-Kutta approximations in control constrained optimal control. SIAM J. Numer. Anal. 38 (2000) 202-226.

[22] R.C. Fetecau, J.E. Marsden, M. Ortiz and M. West, Nonsmooth Lagrangian mechanics and variational collision integrators. SIAM J. Appl. Dyn. Syst. 2 (2003) 381-416.

[23] L. Flatto, Advanced calculus. Williams \& Wilkins (1976).

[24] L. Fox, Some numerical experiments with eigenvalue problems in ordinary differential equations, in Boundary value problems in differential equations, R.E. Langer Ed. (1960).

[25] E. Frazzoli, M.A. Dahleh and E. Feron, Maneuver-based motion planning for nonlinear systems with symmetries. IEEE Trans. Robot. 21 (2005) 1077-1091.

[26] A. Griewank, Evaluating Derivatives: Principles and Techniques of Algorithmic Differentiation. SIAM (2000).

[27] W.W. Hager, Convex control and dual approximations, in Constructive Approaches to Mathematical Models, Academic Press, New York, USA (1979) 189-202.

[28] W.W. Hager, Runge-Kutta methods in optimal control and the transformed adjoint system. Numer. Math. 87 (2000) $247-282$.

[29] W.W. Hager, Numerical analysis in optimal control, in International Series of Numerical Mathematics 139, Birkhäuser Verlag, Basel, Switzerland (2001) 83-93.

[30] E. Hairer, C. Lubich and G. Wanner, Geometric numerical integration. Springer (2002).

[31] S.P. Han, Superlinearly convergent variable-metric algorithms for general nonlinear programming problems. Math. Program. 11 (1976) 263-282.

[32] P. Hiltmann, Numerische Lösung von Mehrpunkt-Randwertproblemen und Aufgaben der optimalen Steuerung über endlichdimensionalen Räumen. Ph.D. Thesis, Fakultät für Mathematik und Informatik, Technische Universität München, Germany (1990).

[33] C.L. Hwang and L.T. Fan, A discrete version of Pontryagin's maximum principle. Oper. Res. 15 (1967) 139-146.

[34] B.W. Jordan and E. Polak, Theory of a class of discrete optimal control systems. J. Elec. Ctrl. 17 (1964) 697-711.

[35] O. Junge and S. Ober-Blöbaum, Optimal reconfiguration of formation flying satellites, in IEEE Conference on Decision and Control and European Control Conference ECC, Seville, Spain (2005).

[36] O. Junge, J.E. Marsden and S. Ober-Blöbaum, Discrete mechanics and optimal control, in 16th IFAC World Congress, Prague, Czech Republic (2005).

[37] O. Junge, J.E. Marsden and S. Ober-Blöbaum, Optimal reconfiguration of formation flying spacecraft - a decentralized approach, in IEEE Conference on Decision and Control and European Control Conference ECC, San Diego, USA (2006) $5210-5215$.

[38] C. Kane, J.E. Marsden and M. Ortiz, Symplectic energy-momentum integrators. Math. Phys. 40 (1999) 3353-3371.

[39] C. Kane, J.E. Marsden, M. Ortiz and M. West, Variational integrators and the Newmark algorithm for conservative and dissipative mechanical systems. Int. J. Numer. Meth. Eng. 49 (2000) 1295-1325.

[40] E. Kanso and J.E. Marsden, Optimal motion of an articulated body in a perfect fluid, in IEEE Conference on Decision and Control and European Control Conference ECC, Seville, Spain (2005). 
[41] W. Karush, Minima of functions of several variables with inequalities as side constraints. Master's thesis, Department of Mathematics, University of Chicago, USA (1939).

[42] H.B. Keller, Numerical methods for two-point boundary value problems. Blaisdell, Waltham, USA (1968).

[43] H.J. Kelley, Gradient theory of optimal flight paths. Journal of the American Rocket Society 30 (1960) 947-953.

[44] L. Kharevych, P. Mullen, S. Leyendecker, Y. Tong, J.E. Marsden and M. Desbrun, Robust time-adaptive integrators for computer animation (in preparation).

[45] M. Kobilarov, Discrete geometric motion control of autonomous vehicles. Ph.D. Thesis, University of Southern California, USA (2008).

[46] M. Kobilarov and G.S. Sukhatme, Optimal control using nonholonomic integrators, in IEEE International Conference on Robotics and Automation (ICRA), Rome, Italy (2007) 1832-1837.

[47] M. Kobilarov, M. Desbrun, J.E. Marsden and G.S. Sukhatme, A discrete geometric optimal control framework for systems with symmetries. Robotics: Science and Systems 3 (2007) 1-8.

[48] D. Kraft, On converting optimal control problems into nonlinear programming problems, in Computational Mathematical Programming F15 of NATO ASI series, K. Schittkowsky Ed., Springer (1985) 261-280.

[49] H.W. Kuhn and A.W. Tucker, Nonlinear programming, in Proceedings of the Second Berkeley Symposium on Mathematical Statisics and Probability, J. Neyman Ed., University of California Press, Berkeley, USA (1951).

[50] T.D. Lee, Can time be a discrete dynamical variable? Phys. Lett. B 121 (1983) 217-220.

[51] T.D. Lee, Difference equations and conservation laws. J. Stat. Phys. 46 (1987) 843-860.

[52] T. Lee, N.H. McClamroch and M. Leok, Attitude maneuvers of a rigid spacecraft in a circular orbit, in American Control Conference, Minneapolis, USA (2006) 1742-1747.

[53] T. Lee, N.H. McClamroch and M. Leok, Optimal control of a rigid body using geometrically exact computations on SE(3), in IEEE CDC and ECC, San Diego, USA (2006) 2710-2715.

[54] D.B. Leineweber, Efficient reduced SQP methods for the optimization of chemical processes described by large sparse DAE models, in Fortschr.-Bericht VDI Reihe 3, Verfahrenstechnik 613, VDI-Verlag (1999).

[55] A. Lew, J.E. Marsden, M. Ortiz and M. West, Asynchronous variational integrators. Arch. Ration. Mech. Anal. 167 (2003) $85-146$.

[56] S. Leyendecker, S. Ober-Blöbaum, J.E. Marsden and M. Ortiz, Discrete mechanics and optimal control for constrained multibody dynamics, in 6th International Conference on Multibody Systems, Nonlinear Dynamics, and Control, ASME International Design Engineering Technical Conferences, Las Vegas, USA (2007).

[57] S. Leyendecker, S. Ober-Blöbaum and J.E. Marsden, Discrete mechanics and optimal control for constrained systems. Optim. Contr. Appl. Meth. (2009) DOI: 10.1002/oca.912.

[58] J.D. Logan, First integrals in the discrete calculus of variation. Aequ. Math. 9 (1973) 210-220.

[59] R. MacKay, Some aspects of the dynamics of Hamiltonian systems, in The dynamics of numerics and the numerics of dynamics, D.S. Broomhead and A. Iserles Eds., Clarendon Press, Oxford, UK (1992) 137-193.

[60] S. Maeda, Canonical structure and symmetries for discrete systems. Math. Jap. 25 (1980) 405-420.

[61] S. Maeda, Extension of discrete Noether theorem. Math. Jap. 26 (1981) 85-90.

[62] S. Maeda, Lagrangian formulation of discrete systems and concept of difference space. Math. Jap. 27 (1981) $345-356$.

[63] J.E. Marsden and S. Shkoller, Multisymplectic geometry, covariant Hamiltonians, and water waves. Math. Proc. Camb. Phil. Soc. 125 (1999) 553-575.

[64] J.E. Marsden and M. West, Discrete mechanics and variational integrators. Acta Numer. 10 (2001) 357-514.

[65] J.E. Marsden, G.W. Patrick and S. Shkoller, Multisymplectic geometry, variational integrators, and nonlinear PDEs. Commun. Math. Phys. 199 (1998) 351-395.

[66] J.E. Marsden, S. Pekarsky and S. Shkoller, Discrete Euler-Poincaré and Lie Poisson equations. Nonlinearity 12 (1999) $1647-1662$.

[67] J.E. Marsden, S. Pekarsky and S. Shkoller, Symmetry reduction of discrete Lagrangian mechanics on Lie groups. Geometry and Physics 36 (1999) 140-151.

[68] J. Martin, Discrete mechanics and optimal control. Master's Thesis, Department of Control and Dynamical Systems, California Institute of Technology, USA (2006).

[69] R.I. McLachlan and S. Marsland, Discrete mechanics and optimal control for image registration, in Computational Techniques and Applications Conference (CTAC) (2006).

[70] A. Miele, Gradient algorithms for the optimization of dynamic systems, in Control and Dynamic Systems 60, C.T. Leondes Ed. (1980) 1-52.

[71] S. Ober-Blöbaum, Discrete mechanics and optimal control. Ph.D. Thesis, University of Paderborn, Germany (2008).

[72] G.W. Patrick and C. Cuell, Error analysis of variational integrators of unconstrained lagrangian systems. Numer. Math. 113 (2009) 243-264.

[73] D. Pekarek, A.D. Ames and J.E. Marsden, Discrete mechanics and optimal control applied to the compass gait biped, in IEEE Conference on Decision and Control and European Control Conference ECC, New Orleans, USA (2007).

[74] L.S. Pontryagin, V.G. Boltyanski, R.V. Gamkrelidze and E.F. Miscenko, The mathematical theory of optimal processes. John Wiley \& Sons (1962). 
[75] M.J.D. Powell, A fast algorithm for nonlinearly constrained optimization calculations, in Numerical Analysis Lecture Notes in Mathematics 630, G.A. Watson Ed., Springer (1978) 261-280.

[76] R. Pytlak, Numerical methods for optimal control problems with state constraints. Springer (1999).

[77] L.B. Rall, Automatic Differentiation: Techniques and Applications, Lect. Notes Comput. Sci. 120. Springer Verlag, Berlin, Germany (1981).

[78] S.D. Ross, Optimal flapping strokes for self-propulsion in a perfect fluid, in American Control Conference, Minneapolis, USA (2006) 4118-4122.

[79] B. Sendov and V.A. Popov, The averaged moduli of smoothness. John Wiley (1988).

[80] Y.B. Suris, Hamiltonian methods of Runge-Kutta type and their variational interpretation. Math. Model. 2 (1990) 78-87.

[81] H. Tolle, Optimization methods. Springer (1975).

[82] O. von Stryk, Numerical solution of optimal control problems by direct collocation, in Optimal Control - Calculus of Variation, Optimal Control Theory and Numerical Methods, R. Bulirsch, A. Miele, J. Stoer and K.H. Well Eds., International Series of Numerical Mathematics 111, Birkhäuser (1993) 129-143.

[83] O. von Stryk, Numerical hybrid optimal control and related topics. Habilitation Thesis, TU München, Germany (2000).

[84] A. Walther, A. Kowarz and A. Griewank, ADOL-C: a package for the automatic differentiation of algorithms written in C/C++. ACM TOMS 22 (1996) 131-167.

[85] J.M. Wendlandt and J.E. Marsden, Mechanical integrators derived from a discrete variational principle. Physica D 106 (1997) 223-246.

[86] J.M. Wendlandt and J.E. Marsden, Mechanical systems with symmetry, variational principles and integration algorithms, in Current and Future Directions in Applied Mathematics, M. Alber, B. Hu and J. Rosenthal Eds., Birkhäuser (1997) $219-261$.

[87] R.E. Wengert, A simple automatic derivative evaluation program. Commun. ACM 7 (1964) 463-464. 